\title{
ARTICLE OPEN Erythropoietin modulates bone marrow stromal cell differentiation
}

\author{
Sukanya Suresh ${ }^{1}$, Luis Fernandez de Castro ${ }^{2}$, Soumyadeep Dey ${ }^{1}$, Pamela G. Robey ${ }^{2}$ and Constance Tom Noguchi ${ }^{1}$
}

Erythropoietin is essential for bone marrow erythropoiesis and erythropoietin receptor on non-erythroid cells including bone marrow stromal cells suggests systemic effects of erythropoietin. Tg6 mice with chronic erythropoietin overexpression have a high hematocrit, reduced trabecular and cortical bone and bone marrow adipocytes, and decreased bone morphogenic protein 2 driven ectopic bone and adipocyte formation. Erythropoietin treatment ( $\left.1200 \mathrm{IU} \cdot \mathrm{kg}^{-1}\right)$ for 10 days similarly exhibit increased hematocrit, reduced bone and bone marrow adipocytes without increased osteoclasts, and reduced bone morphogenic protein signaling in the bone marrow. Interestingly, endogenous erythropoietin is required for normal differentiation of bone marrow stromal cells to osteoblasts and bone marrow adipocytes. $\triangle \mathrm{EpoR}_{\mathrm{E}}$ mice with erythroid restricted erythropoietin receptor exhibit reduced trabecular bone, increased bone marrow adipocytes, and decreased bone morphogenic protein 2 ectopic bone formation. Erythropoietin treated $\Delta E p o R_{E}$ mice achieved hematocrit similar to wild-type mice without reduced bone, suggesting that bone reduction with erythropoietin treatment is associated with non-erythropoietic erythropoietin response. Bone marrow stromal cells from wild-type, $\mathrm{Tg} 6$, and $\Delta$ EpoR $_{\mathrm{E}}$-mice were transplanted into immunodeficient mice to assess development into a bone/marrow organ. Like endogenous bone formation, Tg6 bone marrow cells exhibited reduced differentiation to bone and adipocytes indicating that high erythropoietin inhibits osteogenesis and adipogenesis, while $\Delta$ Epo $R_{E}$ bone marrow cells formed ectopic bones with reduced trabecular regions and increased adipocytes, indicating that loss of erythropoietin signaling favors adipogenesis at the expense of osteogenesis. In summary, endogenous erythropoietin signaling regulates bone marrow stromal cell fate and aberrant erythropoietin levels result in their impaired differentiation.

Bone Research (2019)7:21; https://doi.org/10.1038/s41413-019-0060-0

\section{INTRODUCTION}

Erythropoietin (EPO), a cytokine produced in fetal liver and adult kidneys, is required for production of red blood cells. EPO binds to its receptor, EPOR, expressed on erythroid progenitors and promotes survival, proliferation, and differentiation. ${ }^{1}$ Mice with targeted deletion of Epo or Epor die in utero of severe anemia. ${ }^{2,3}$ Recombinant human EPO is clinically used to treat anemia resulting from chronic kidney disease and chemotherapy. ${ }^{4}$ Functional EPOR is present in endothelial cells, ${ }^{5}$ neurons, ${ }^{6}$ skeletal muscle progenitor cells, ${ }^{7}$ adipocytes, ${ }^{8}$ and islets ${ }^{9}$ suggesting that endogenous EPO signaling exerts systemic regulation and that EPO administered in patients could interact with EPOR in nonerythroid cells with implications beyond erythropoiesis. To understand the full spectrum of clinical utility of EPO, studies to elucidate EPO response in non-erythroid cells are required.

Bone marrow contains two distinct types of stem cells: hematopoietic stem cells that give rise to all types of blood cells and skeletal stem cells (SSCs), a subset of bone marrow stromal cells (BMSCs) that differentiate into chondrocytes, osteoblasts, hematopoiesis supporting stroma and adipocytes. ${ }^{10}$ Lineage commitment and differentiation of SSCs/BMSCs depends on interplay of transcription factors and signaling molecules. Among several transcription factors regulating SSC/BMSC differentiation, PPAR- $\gamma$, CEBP- $\alpha$, and CEBP- $\beta$ enhance adipogenesis, whereas
RUNX2 and OSTERIX are important for osteogenesis. Several cytokines present in the bone marrow niche also preferentially regulate SSC/BMSC differentiation. ${ }^{11}$ Impaired SSC/BMSC differentiation results in imbalance of adipocyte and osteoblast differentiation in marrow and clinical studies show expansion of marrow fat is associated with reduced bone density. ${ }^{12,13}$ Enhanced marrow adipogenesis and bone fragility are also observed in osteoporosis, ${ }^{14}$ obesity, ${ }^{15}$ and diabetes. ${ }^{16}$

Bone is metabolically active and undergoes continuous remodeling processes whereby osteoclasts of hematopoietic origin digest old bone and osteoblasts of BMSC origin lay down new bone matrix. ${ }^{17}$ EPO has been shown to promote the differentiation of these cells in in vitro culture conditions, ${ }^{18}$ although lineage tracing using Epor-Cre knock-in mice showed labeling only in cells of premegakaryocyte/erythroid origin in the bone marrow microenvironment. ${ }^{19}$ In fracture healing models, EPO demonstrated a beneficial effect on bone $e^{20,21}$ and accelerated wound healing, and supraphysiologic EPO promoted bone growth in vertebrae of young and old mice. ${ }^{18}$ Exogenous EPO treatment in animal models induced low bone mass in long bones, ${ }^{22}$ indicating that skeletal response to EPO depends on location of the bone, EPO dose, and extent of bone injury. Here, we show that EPOR is expressed on BMSCs and that high EPO inhibits osteogenesis and adipogenesis using transgenic mice with EPO

\footnotetext{
${ }^{1}$ Molecular Medicine Branch, National Institute of Diabetes and Digestive and Kidney Diseases, National Institutes of Health, Bethesda, MD 20892, USA and ${ }^{2}$ Skeletal Biology Section, National Institute of Dental and Craniofacial Research, National Institutes of Health, Bethesda, MD 20892, USA

Correspondence: Constance Tom Noguchi (connien@niddk.nih.gov)
} 

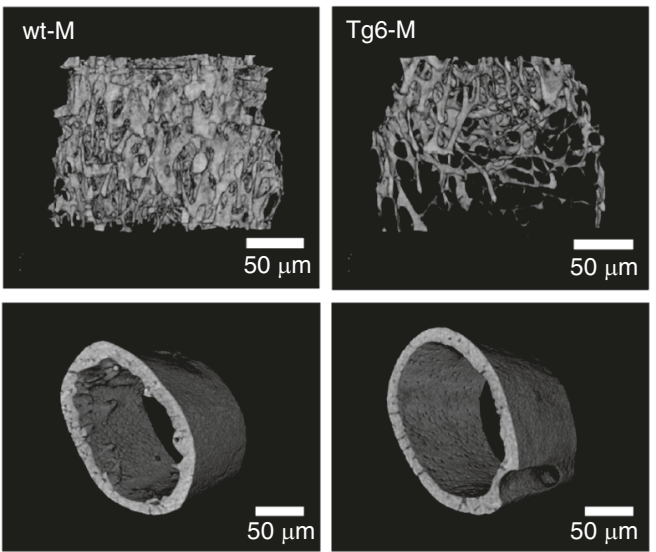

e

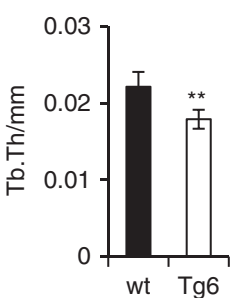

$\mathbf{f}$

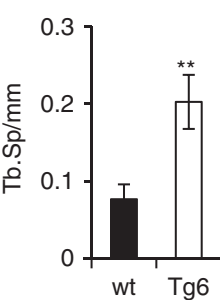

g

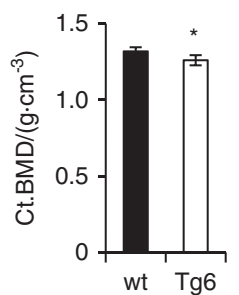

b

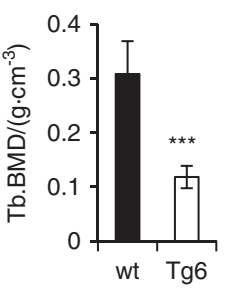

h

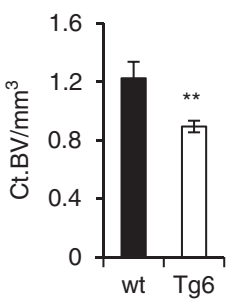

c

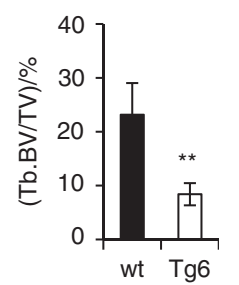

i

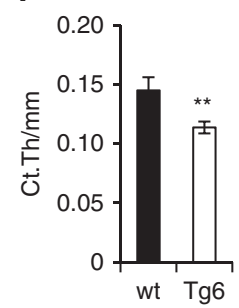

d

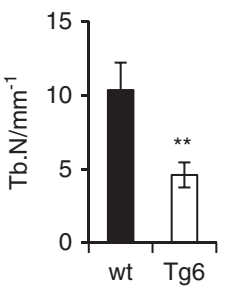

j

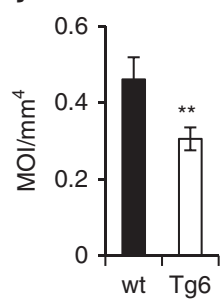

Fig. 1 Bone features of Tg6 mice overexpressing EPO. a Micro-CT generated 3D images of trabecular (top) and cortical bone (bottom) of the femurs of 11-week-old wild-type (wt; left) and Tg6 mice (right). b-f Quantification of trabecular parameters including trabecular bone mineral density (BMD), bone volume/total volume (BV/TV), number (N), thickness (Th), and spacing (Sp) of trabeculae. g-j Quantification of cortical BMD, BV, thickness, and moment of inertia (MOI) $\left(n=4 /\right.$ group, ${ }^{*} P<0.05$, ${ }^{* *} P<0.01$, $\left.{ }^{* * *} P<0.001\right)$

overexpression $(\mathrm{Tg} 6)^{23}$ that exhibit increased hematocrit, reduced bone, and reduction in bone marrow adipocytes. We also show that endogenous EPO regulates bone homeostasis and marrow adipogenesis using transgenic mouse with Epor expression restricted to erythroid cells $\left(\triangle \mathrm{EpoR}_{\mathrm{E}}\right)^{24}$ in which lack of EPOR signaling in SSCs/BMSCs disrupts their differentiation to promote adipogenesis and decrease osteogenesis.

\section{RESULTS}

Chronic elevated EPO levels reduces bones in mice

To analyze the effect of elevated EPO on bone architecture, we used the transgenic Tg6 mice with overexpression of human EPO. The Tg6 mice have mouse EPO levels similar to their littermate controls but have high levels of human EPO in their circulation (Supplementary Fig. 1b), similar to earlier reports. ${ }^{23}$ Micro-Ct scans (Fig. 1a) showed $61 \%$ reduced trabecular bone mineral density (BMD) (Fig. 1b) and 63\% reduced trabecular bone volume/tissue volume (BV/TV) (Fig. 1c) in 11-week-old male Tg6 mice compared with littermate controls. Tg6 mice also had reduced trabecular number (Fig. 1d), thickness (Fig. 1e), and increased trabecular spacing (Fig. 1f). Analysis of cortical bone parameters of Tg6 mice showed reduced cortical BMD, (Fig. 1g) BV (Fig. 1h), thickness (Fig. 1i), and moment of inertia (Fig. 1j). We compared femur length and body weight of male and female Tg6 mice and found that only male Tg6 mice had shorter femurs (Supplementary Fig. 2a) and lower body mass (Supplementary Fig. 2b). Female Tg6 mice were not different in these parameters compared with female littermate controls.

Mice with erythroid-restricted Epor expression have reduced bone histomorphometric parameters

We used transgenic $\Delta$ EpoR $_{E}$-mouse model with Epor expression restricted to erythroid cells to assess the role of endogenous EPO signaling in regulation of osteogenic cells. Consistent with the previous report, ${ }^{24}$ we observed similar mouse EPO levels in the serum of $\Delta$ EpoR $_{E}$ mice and wild-type controls (Supplementary Fig. 1a). Micro-Ct measurements of 11-week-old male $\Delta$ EpoR $_{E}$ mice (Fig. 2a) showed $40 \%$ reduced trabecular $\mathrm{BMD}, 40.5 \%$ reduced trabecular BV/TV, $22.8 \%$ reduced trabecular number, and $45.2 \%$ increase in trabecular spacing (Fig. 2b). Female $\triangle E_{p o R_{E}}$ mice also exhibited reduced trabecular bone with $29.8 \%$ reduced $B M D, 40 \%$ reduced trabecular BV/TV, 30.6\% fewer trabeculae, and $51.2 \%$ increase in trabecular spacing compared to wild-type female mice (Fig. 2c). There was no significant difference in cortical bone of both male and female $\Delta E_{E p o R_{E}}$ mice compared with control mice (Supplementary Fig. 2e, f). Femoral length was also not different in $\triangle \mathrm{EpoR}_{\mathrm{E}}$ mice compared with wild-type mice (Supplementary Fig. 2c). Male and female $\Delta$ EpoR $_{E}$ mice had increased body mass (Supplementary Fig. 2d), consistent with previous reports. ${ }^{8}$ To determine if reduced bone mass seen with $\Delta E p o R_{E}$ mice is due to increased body weight, age and weight-matched female wild-type mice were compared with female $\Delta E p o R_{E}$ mice. In female $\Delta E p o R_{E}$ mice, reduced trabecular bone was extensive (Supplementary Figs. 2g, h, i) and cortical BV was lower (Supplementary Fig. 2j) compared with age and weight-matched control mice.

Elevated EPO does not reduce bone parameters in mice with Epor deletion in non-erythroid cells

To determine whether reduction in bone with high EPO is associated with EPO-stimulated erythropoiesis or mediated by non-erythroid cells, we treated young wild-type and $\triangle E p o R_{E}$ mice (8-week-old females) with EPO (1 $200 \mathrm{lU} \cdot \mathrm{kg}^{-1}$ ) for 10 days (Fig. 2d). EPO treatment increased hematocrit similarly in wild-type and $\triangle$ EpoR $_{\mathrm{E}}$ mice from $49 \% \pm 1.36 \%$ and $49.5 \% \pm 1.36 \%$ to $68.4 \% \pm$ $2.23 \%$ and $70.4 \% \pm 1.51 \%$, respectively. EPO treatment reduced trabecular BMD (-22\%) (Fig. 2e), BV/TV (-26.6\%) (Fig. 2f), trabecular number (-25\%) (Fig. 2g), and increased trabecular spacing (39.5\%) (Fig. 2h) in wild-type mice, but did not induce any changes in the bones of $\triangle E p o R_{E}$ mice. Body weight of wild-type 

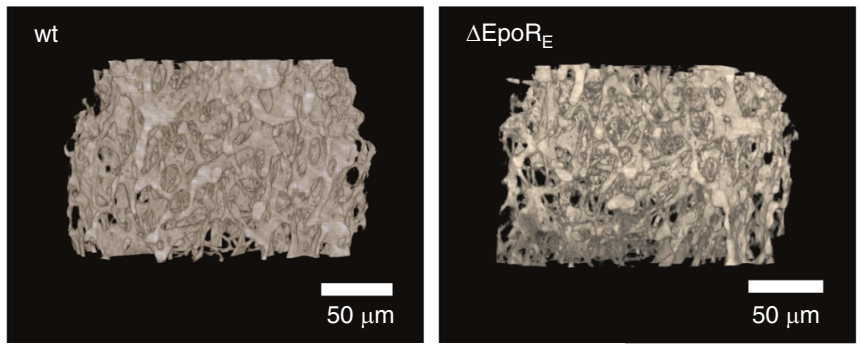

b
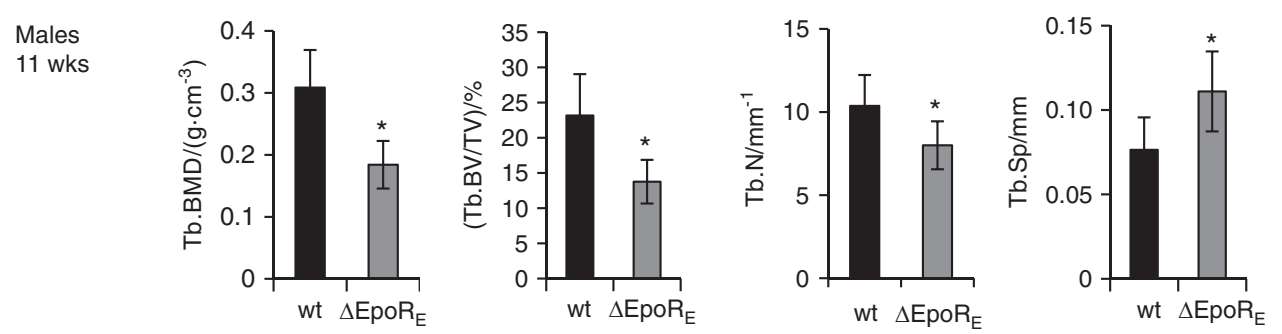

C Females
11 wks
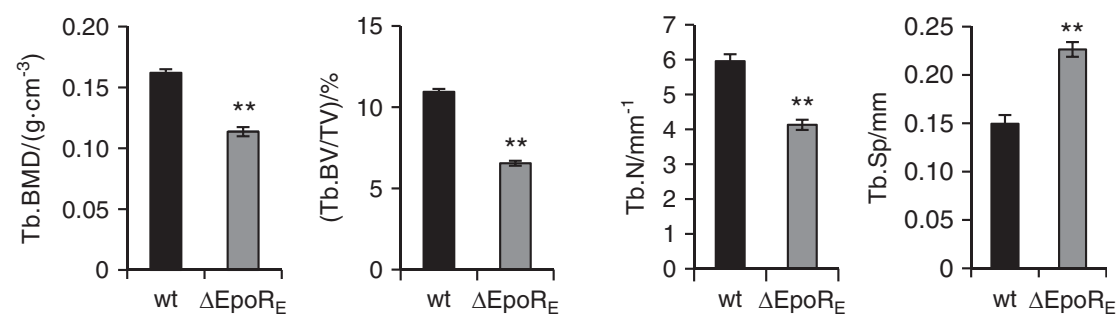

d

8 wks
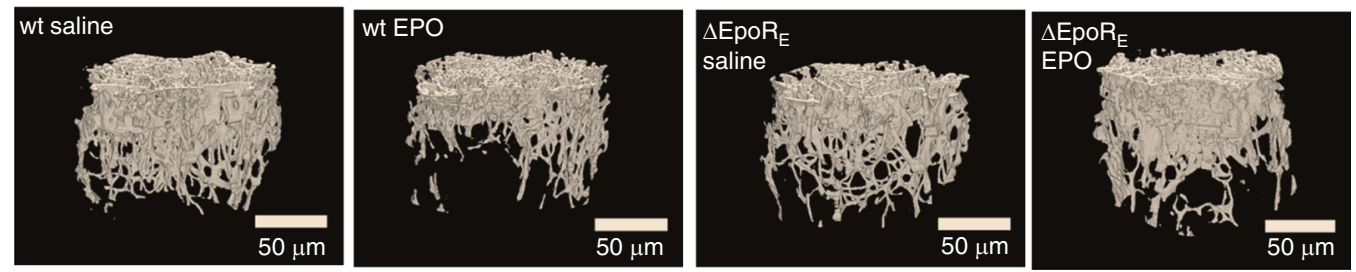

e

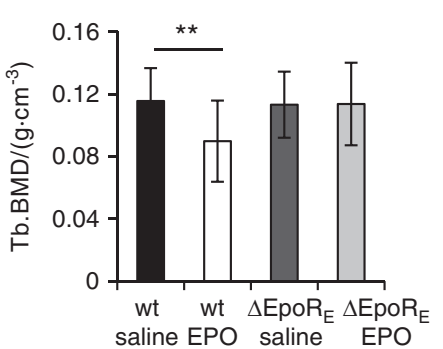

f

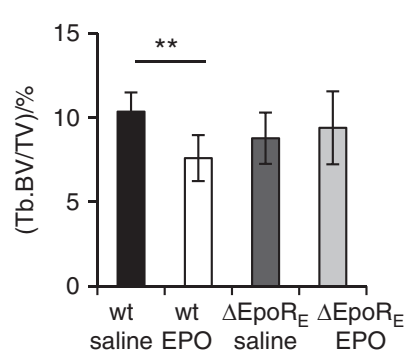

g

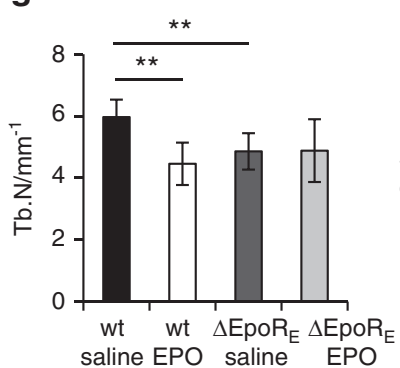

h

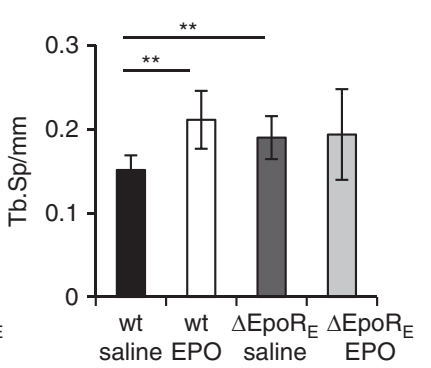

Fig. 2 Bone features of $\triangle E$ EpoR $\mathrm{R}_{\mathrm{E}}$ mice with lack of EPOR signaling in non-erythroid cells. a Micro-CT generated images of trabecular bone of 11-week-old male wild-type (wt) and $\Delta \mathrm{EpoR}_{\mathrm{E}}$ mice. $\mathbf{b}$-c Femurs of 11-week-old male and female $\Delta \mathrm{Eppo}_{\mathrm{E}}$ mice and age matched wt mice were analyzed using micro-CT. Quantitation of trabecular BMD, BV/TV, number, and spacing of trabecular bone of male (b) and female $\Delta E$ EpoR $R_{E}$ mice (c) and controls ( $n=4 /$ group). d-h EPO induced changes in bones of 8-week-old wild-type (wt) and $\Delta$ EpoR $\mathrm{E}_{\mathrm{E}}$ mice with Epor deletion in nonerythroid cells. d 3D images of trabecular bones of wt and $\triangle E$ EpoR $R_{E}$ mice receiving $1200 \mathrm{IU} \cdot \mathrm{kg}^{-1}$ of EPO for 10 days. Quantification of trabecular parameters including trabecular BMD (e), BV/TV (f), trabecular number (g), and trabecular spacing (h). ( $\left(n=5 /\right.$ group, ${ }^{*} P<0.05,{ }^{* *} P<0.01,{ }^{* * *} P<$ $0.001)$

and $\triangle \mathrm{EpoR}_{\mathrm{E}}$-mice with and without EPO treatment was similar (data not shown), excluding the influence of body weightassociated changes in bone parameters. Compared with wildtype control mice, $\triangle E p o R_{E}$ mice not treated with EPO showed reduced trabecular number (-18.5\%) (Fig. 2g) and increased trabecular spacing (25.5\%) (Fig. 2h).
Bone marrow adipocytes are responsive to EPO

To assess EPO influence on the bone marrow fat depot, we analyzed H\&E stained femur sections of Tg6 mice (11 weeks old) and their littermate controls (Fig. 3a, d). Both male and female Tg6 mice showed extensive loss of marrow adipocytes. In contrast, analysis of femur sections of male and female $\Delta$ EpoR $_{E}$ mice 
a 11 wks
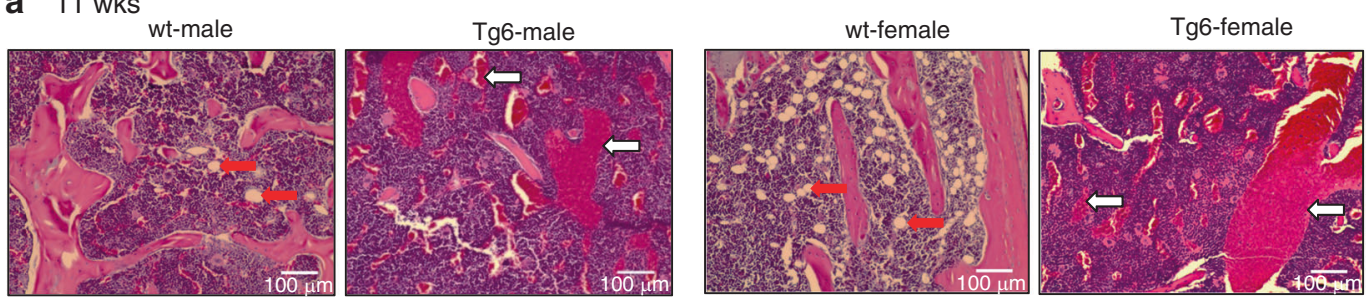

b 11 wks
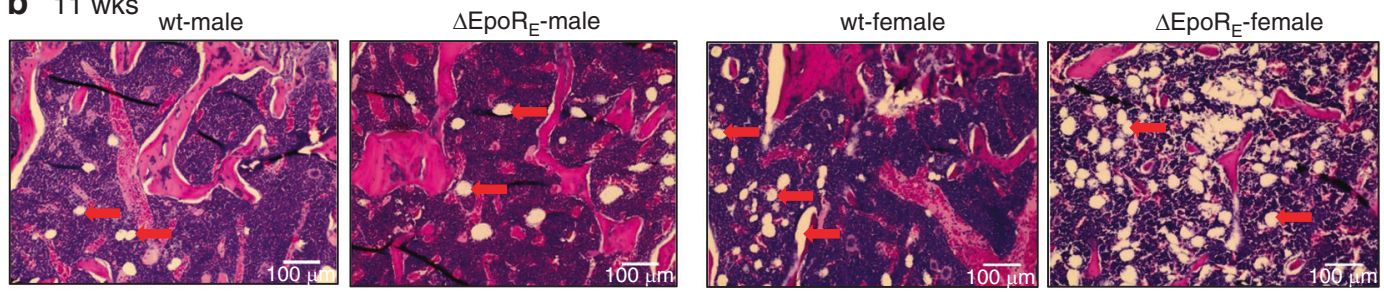

C $8 w k s$
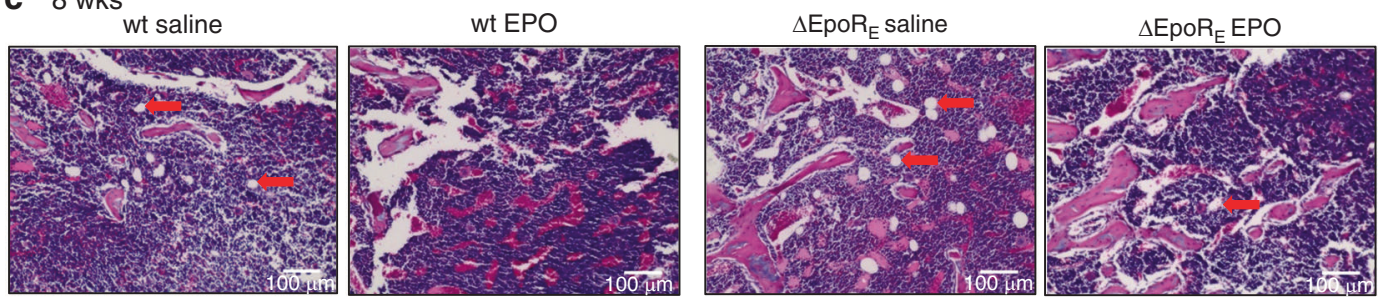

d 11 wks

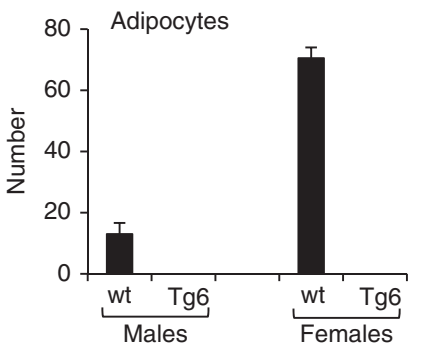

e 11 wks

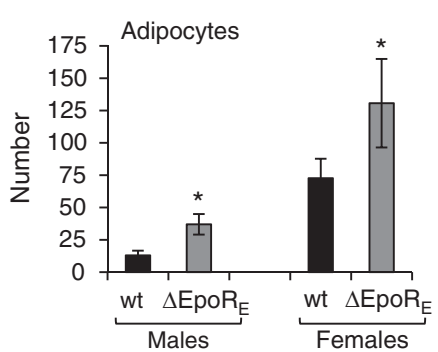

f $8 w k s$

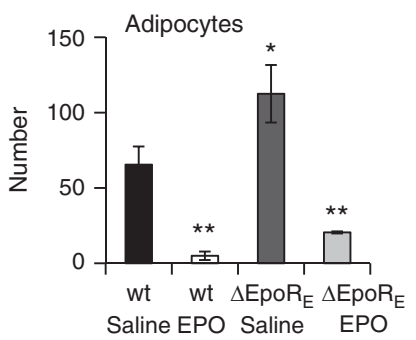

Fig. 3 Histology analysis by $\mathrm{H} \& \mathrm{E}$ staining of femurs sections of Tg6- and $\Delta \mathrm{EpoR}_{\mathrm{E}}$-mice. a Femurs of 11-week-old male and female Tg6 mice and controls. Red arrows indicate marrow adipocytes, which appear as white circles in the marrow, pink colored regions are bones and blue regions are the marrow (BM). White arrows in Tg6 histology images show sinusoids ( $n=4 /$ group). b Femurs of 11 -week-old male and female $\Delta \mathrm{EpoR}_{\mathrm{E}}$ mice and controls ( $n=4$ /group). c Femur sections of 8-week-old wt and $\Delta \mathrm{EpoR}$ mice receiving $1200 \mathrm{IU} \cdot \mathrm{kg}^{-1}$ of EPO for ten days. d Number of marrow adipocytes in femurs of male and female Tg6 mice ( $n=5 / g r o u p)$. e Number of marrow adipocytes in the femurs of wildtype and $\Delta E p o R_{E}$ mice. $f$ Number of adipocytes in the femurs of wild-type and $\Delta E p o R_{E}$ mice receiving $1200 \mathrm{IU} \cdot \mathrm{kg}^{-1}$ of EPO or saline treatment for ten days. ${ }^{*} P<0.05,{ }^{* *} P<0.01,{ }^{* * *} P<0.001$

showed increased marrow adipocytes (Fig. 3b, e). In both wildtype and $\triangle \mathrm{EpoR}_{\mathrm{E}}$ groups, female mice had significantly more marrow adipocytes than age-matched male mice. We next investigated changes in bone marrow adipocytes with exogenous administration of EPO. Both wild-type and $\triangle E_{p o R_{E}}$ mice (8 weeks old, female) treated with EPO showed reduced bone marrow adipocytes compared with saline treatment (Fig. 3c, f).

Effect of EPO signaling on osteogenic and osteoclast differentiation in vitro

We performed in vitro differentiation assays to determine if chronically elevated EPO has a direct effect on osteogenic cells and osteoclasts. Long-term cultures of calvarial osteogenic cells in osteogenic medium (Fig. 4a) showed that Tg6-osteogenic cells having increased mineralization potential along with increased $A / p$ and Osterix expression with no significant changes in Runx2 (Fig. 4b). Tg6-osteogenic cells also expressed human EPO, although Epor levels were similar to wild-type osteogenic cells
(Supplementary Fig. 3a, b). High EPO levels in these cells did not affect their viability (Fig. 4c). Except for a transient decrease in ALP activity measured at $48 \mathrm{~h}$ in osteogenic cultures from Tg6 mice, no significant differences in ALP activity were observed (Fig. 4d).

Primary calvarial osteogenic cells of $\Delta$ EpoR $_{E}$ mice cultured in osteogenic medium showed lower differentiation and mineralization as identified by ALP and alizarin red staining, respectively (Fig. 4e). Gene expression analysis showed less Alp expression, but no changes in Runx2 and Osterix levels in $\triangle \mathrm{EpoR}_{\mathrm{E}}$-Osteogenic cells cultured for 7 days in osteogenic medium (Fig. 4f). Addition of EPO to in vitro cultures of wild-type osteogenic cells resulted in a transient increase in viability along with reduction in their differentiation potential at $24 \mathrm{~h}$ as evidenced by decreased ALP activity (Fig. 4g, h). However, long-term calvarial osteogenic cultures of wild-type mice with EPO addition did not show any effect in their differentiation and mineralization ability (data not shown). The osteogenic cells from $\triangle E p o R_{E}$ mice had a reduced differentiation ability compared with wild-type cells. Reduced Epor 

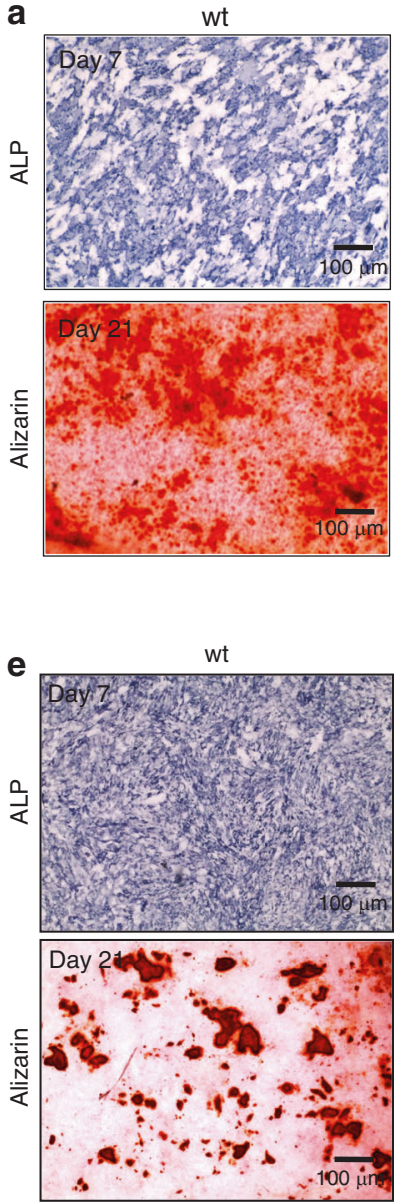

g

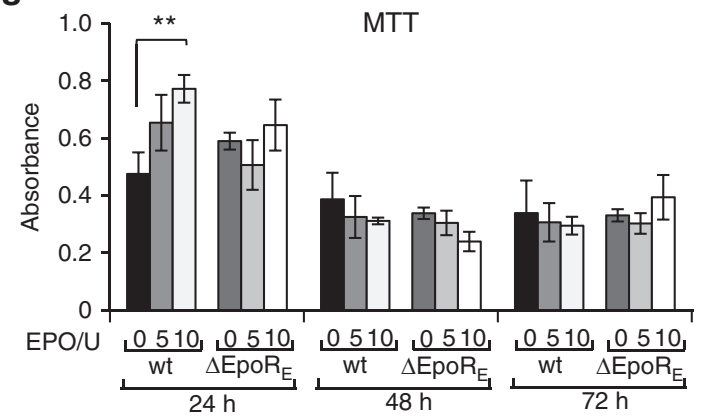

b
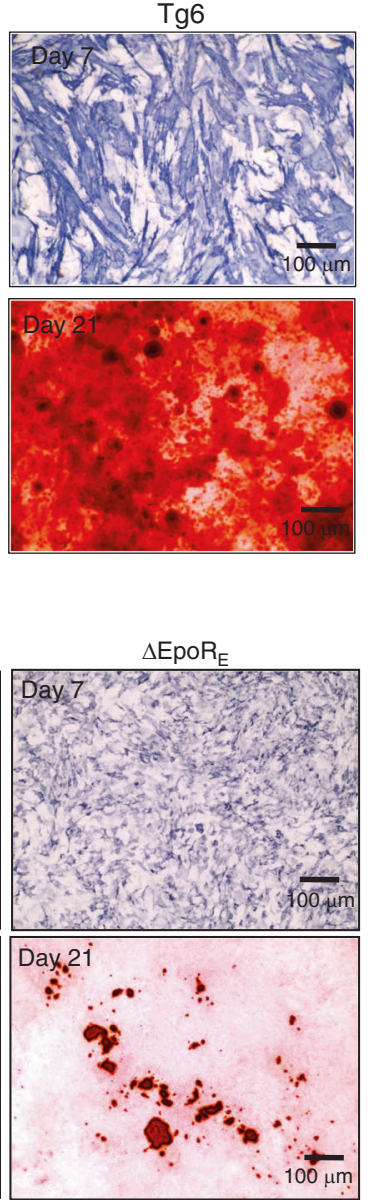

f
Day 7
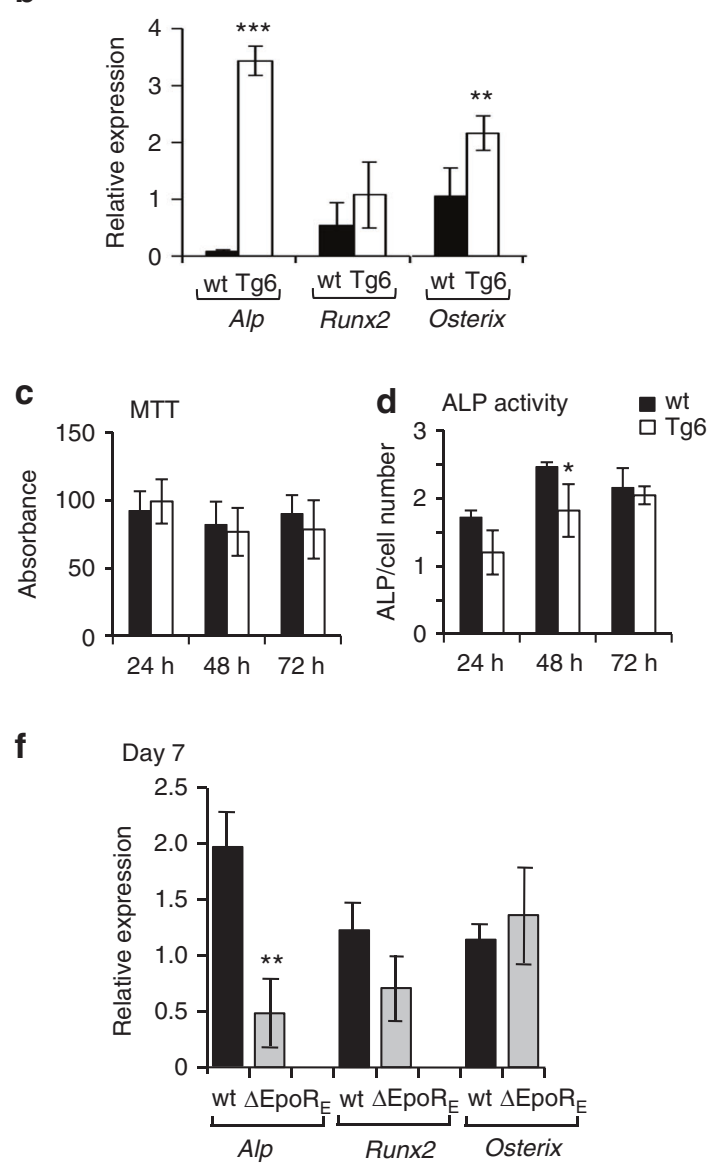

h

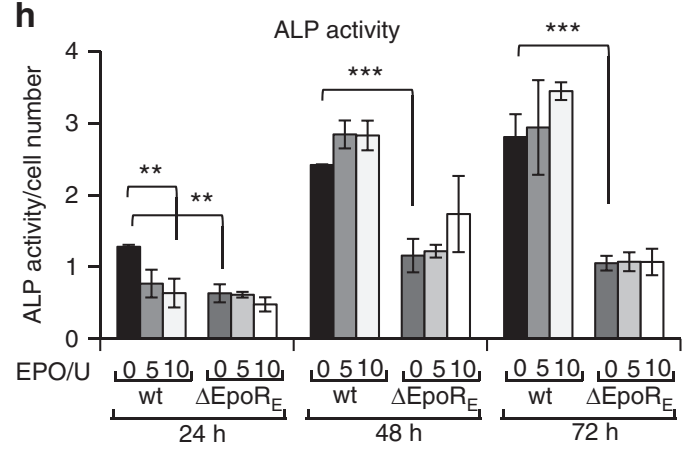

Fig. 4 Osteogenic cultures of Tg6- and $\Delta E_{\text {EpoR }}$-mice. a Calvarial osteoprogenitors isolated from wt and Tg6-mice were cultured in osteogenic medium and stained for ALP expression on day 7 and mineralization deposits by alizarin red staining on day 21. b Relative expression of Alp, Runx2 and Osterix in osteogenic cultures on day 7 determined by real-time PCR. c Cell proliferation of calvarial osteoblasts was determined using MTT assay at 24,48 , and $72 \mathrm{~h}$ of culture in osteogenic medium and data expressed as absorbance values. $\mathbf{d}$ ALP activity was measured using a colorimetric assay and expressed as absorbance normalized to the cell number determined by MTT assay. e Calvarial osteoprogenitors isolated from wt and $\Delta$ EpoR $_{\mathrm{E}}$-mice cultured in osteogenic medium and stained for ALP (day 7) and alizarin red (day 21). $\mathbf{f}$ Relative expression of Alp, Runx2 and Osterix mRNA levels from primary calvarial osteogenic cells (day 7) determined by real-time PCR. $\mathbf{g}$-h Calvarial osteogenic cells from wt and $\triangle E_{\text {EpoR }}$-mice treated with $5 \mathrm{U}$ and $10 \mathrm{U}$ of EPO and measured for their cell proliferation rate by MTT assay (g) and ALP activity of the cells were measured using colorimetric assays, absorbance was normalized to the cell number (h). ( $n=4 /$ group, ${ }^{* *} P<0.01,{ }^{* * *} P$ $<0.001)$

expression was detected on $\Delta$ Epo $_{\mathrm{E}}$-Osteogenic cells (Supplementary Fig. 3c), and they did not respond to EPO treatment (Fig. $4 \mathrm{~g}$, h). These data suggest a direct effect of EPO on osteogenic cells. These observations are also consistent with the in vivo observations of reduced trabecular bone seen in $\triangle E_{\text {EpoR }}$ mice (Fig. 2) and unchanged bone architecture seen with EPO treatment in these mice (Fig. 2d-h).
Femurs of Tg6 mice analyzed by micro-Ct (Fig. 1) were stained for TRAP to detect osteoclasts and EPO overexpression in Tg6 mice was associated with increased osteoclasts (Fig. 5a, e). Bone marrow derived osteoclast cultures showed Tg6-cells formed numerous giant multinucleated osteoclasts (Fig. 5b, f). Tg6-osteoclasts expressed human EPO, but reduced Epor on day 4 of differentiation compared with wild-type osteoclasts (Supplementary Fig. 3d, e). 
a 11 wks

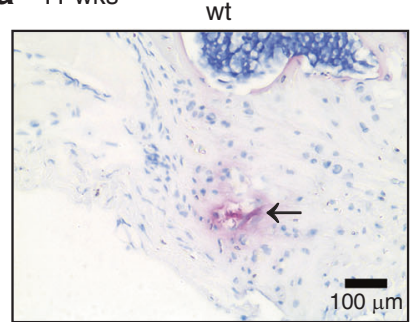

C 11 wks

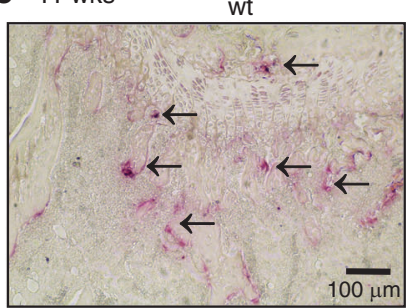

e

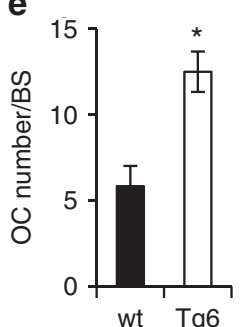

Tg6

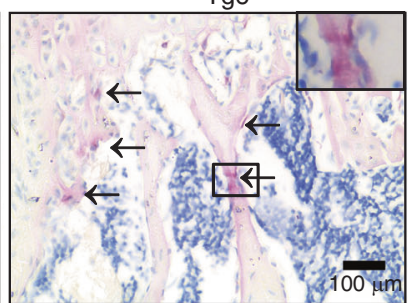

$\Delta \mathrm{EpoR}_{\mathrm{E}}$
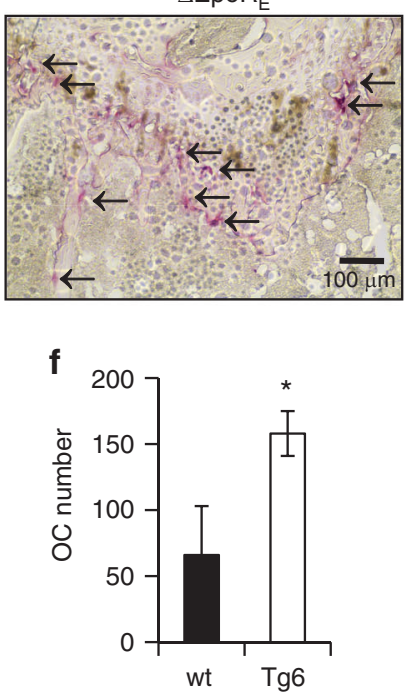

b

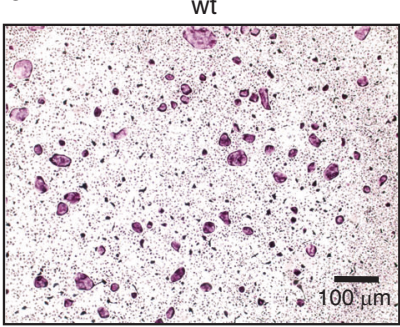

d
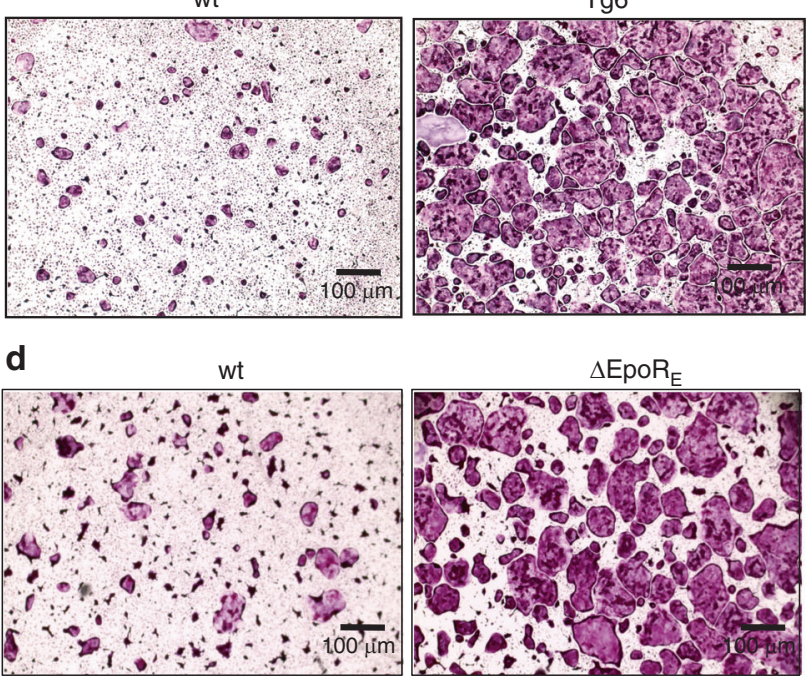

$\Delta \mathrm{EpoR}_{\mathrm{E}}$

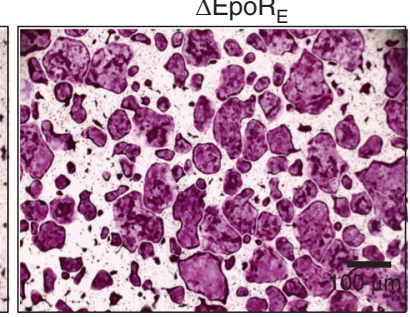

g

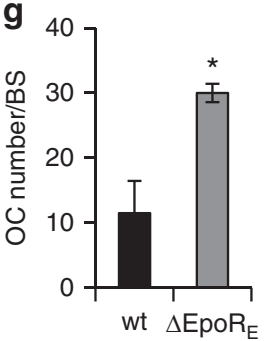

h

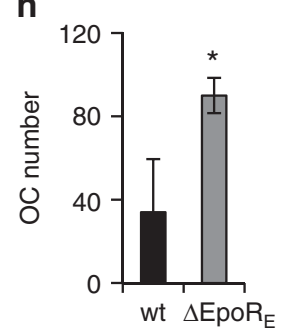

i 8 wks

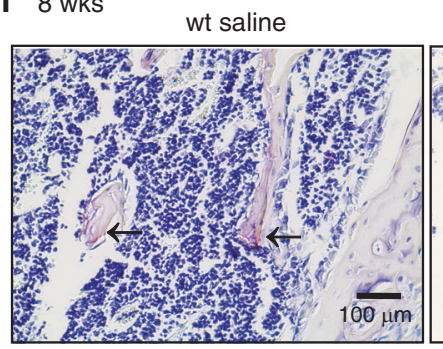

wt EPO

$\Delta$ EpoR $_{E}$ saline

$\triangle \mathrm{EpoR}_{\mathrm{E}} \mathrm{EPO}$
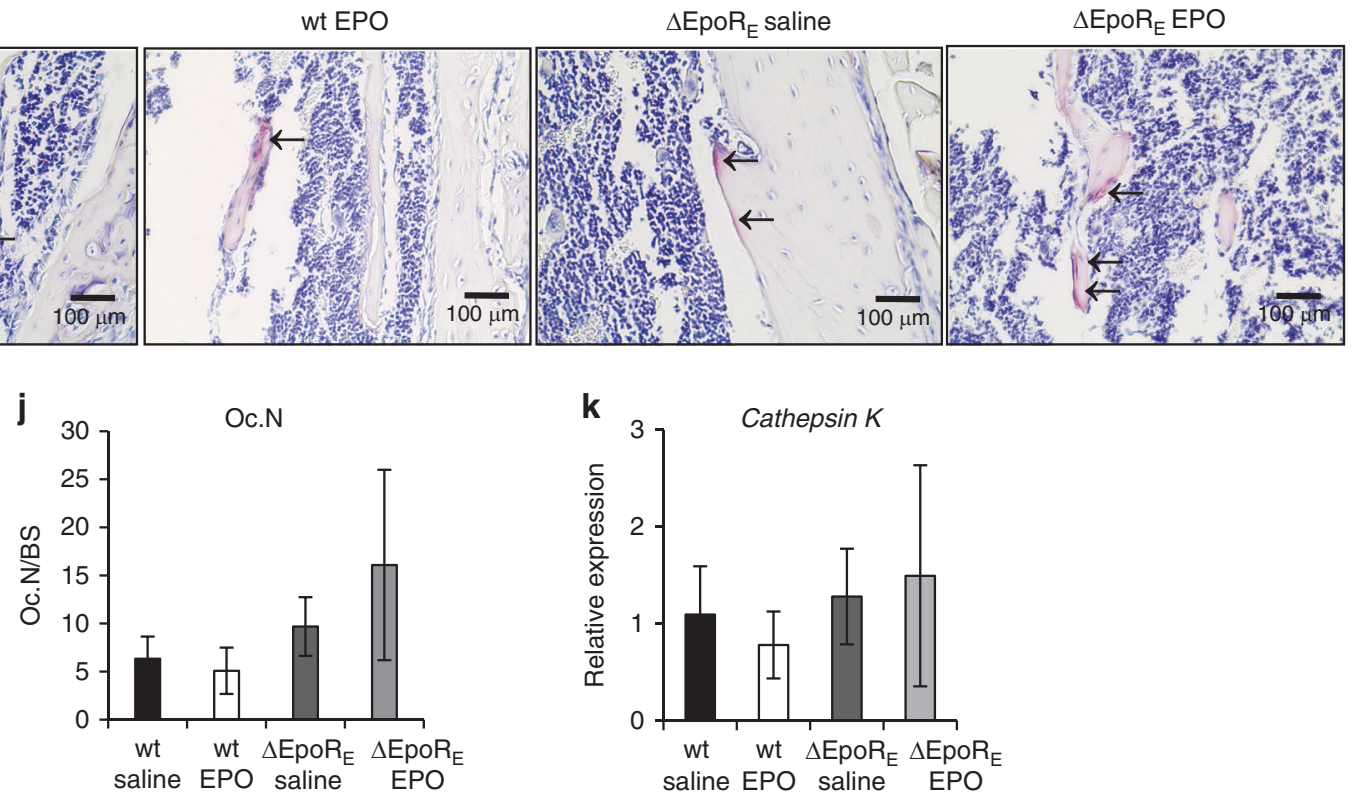

Fig. 5 Osteoclasts in Tg6- and $\triangle$ EpoR $_{E}$ mice. a Representative images of TRAP stained osteoclasts in the femur sections of 11-week-old wt and Tg6-mice. Arrows indicate purple stained osteoclasts along the surface of trabecular bones. $\mathbf{b}$ Images of in vitro osteoclast differentiation of bone marrow cells of wild-type (wt) and Tg6-mice using M-CSF and RANKL. Osteoclasts appear after 4-5 days and were stained for TRAP. c TRAP-stained sections of femurs of 11-week-old wild-type (wt) and $\Delta E_{\text {Epo }} \mathrm{R}_{\mathrm{E}}$-mice showing osteoclasts. Arrows indicate purple stained osteoclasts along the surface of trabecular bone. $\mathbf{d}$ In vitro osteoclast differentiation from the bone marrow of wt and $\Delta$ EpoR $_{\mathrm{E}}-\mathrm{mice}$ identified by TRAP staining. e-h Number of osteoclasts on the bone surface of wt and Tg6-mice (e), number of osteoclasts in the bone marrow cultures of wt and Tg6-mice (f), number of osteoclasts on the bone surface of wt and $\Delta$ EpoR $_{\mathrm{E}}-\mathrm{mice}(\mathbf{g})$, and number of osteoclasts in the bone marrow cultures of wt and $\Delta$ EpoR $_{E}-$ mice (h) analyzed using ImageJ. i TRAP stained femur sections of 8-week-old wt and $\Delta$ EpoR $R_{E}-m i c e$ receiving $1200 \mathrm{U} \cdot \mathrm{kg}^{-1}$ of EPO or saline for 10 days. Arrows indicate purple stained osteoclasts along the trabecular bone surface. $\mathrm{j}$ Enumeration of osteoclasts in the wt and $\triangle \mathrm{EpoR}_{\mathrm{E}}$-mice receiving EPO or saline. $\mathbf{k}$ Relative expression of Cathepsin $\mathrm{K}$ levels in the whole bone marrow determined by real-time PCR. $\left(n=5 /\right.$ group, ${ }^{*} P<0.05$, ${ }^{* *} P<0.01$, $\left.{ }^{* * *} P<0.001\right)$ 
Therefore, chronic EPO overexpression in Tg6 mice accelerates differentiation of osteoclasts and osteoblasts in vitro suggesting a high bone turnover rate in Tg6 mice. ${ }^{19}$

TRAP stained sections of femurs of 11 -week-old $\Delta$ EpoR $_{E}-$ mice showed increased osteoclasts lining the bone surface (Fig. 5c, g). Consistent with in vivo observations, we found numerous multinucleated osteoclasts in bone marrow cultures of $\triangle E p o R_{E}$ mice (Fig. $5 \mathrm{~d}$, h). Osteoclasts of $\Delta$ EpoR $_{E}$ mice expressed Epor comparable to wild-type osteoclasts (Supplementary Fig. 3f). Since, the Epor expression in $\triangle E p o R_{E}$ mice is Gata1 dependent, we measured Gata1 mRNA levels in FACS sorted preosteoclasts from the bone marrow and found both wild-type and $\triangle E$ Eo $R_{E}$-mice preosteoclasts expressing Gata1 (Supplementary Fig. 3h). EPO treatment of bone marrow cultures from wild-type and $\Delta$ Epo $_{E}$-mice had no effect on their differentiation (Supplementary Fig. 3g). However, unlike 11-week-old $\Delta E p o R_{E}$ mice, young $\Delta E p o R_{E}$ mice of 8 weeks of age did not have any increase in osteoclasts in bone sections (Fig. $5 \mathrm{i}$, j). Compared to age-matched wild-type mice, $\Delta \mathrm{EpoR}_{\mathrm{E}}$ mice gain increased fat mass and body weight with age and exhibit systemic inflammation ${ }^{25}$ as confirmed by significantly high Creactive protein in the serum (Supplementary Fig. 1c). Therefore, the increase in osteoclasts seen in 11-week-old mice might be associated with high level of inflammation occurring in these mice with body weight gain. We did not observe any increase in osteoclasts in EPO treated wild-type mice (Fig. 5i, j). Whole bone marrow analysis showed no differences in Cathepsin $K$ expression, a bone resorption marker secreted by osteoclasts (Fig. 5k).

Elevated EPO inhibits bone morphogenetic protein 2 (BMP2)induced bone formation

Since acute EPO treatment reduced bone without apparent changes in osteoclasts, we focused on the role of EPO in osteogenic differentiation. BMPs promote osteogenic differentiation; therefore, we assessed levels of several BMPs in whole bone marrow of wild-type, Tg6-, and $\Delta E \mathrm{EpoR}_{\mathrm{E}}$-mice, and mice treated with EPO. Among the BMPs, we detected Bmp2 and Bmp6 in whole bone marrow and found Tg6 mice with chronic EPO had reduced Bmp2 and Bmp6 expression (Supplementary Fig. 4a, b). Elevated EPO also decreased Bmp2 and Bmp6 in bone marrow of wild-type mice, but not in $\triangle \mathrm{EpoR}_{\mathrm{E}}$ mice. However, saline treated $\triangle \mathrm{EpoR}_{\mathrm{E}}$ mice had low Bmp2 levels in bone marrow (Supplementary Fig. 4c, d).

BMP2 is a potent osteogenic factor ${ }^{26}$ and has also been shown to promote adipogenesis. ${ }^{27,28}$ To probe EPO-BMP2 interaction in bone development, a BMP2-induced ectopic bone formation assay was performed by subcutaneous implantation of collagen sponges soaked with $5 \mu \mathrm{g}$ of BMP2 into Tg6 mice, wild-type littermates, $\triangle E_{p o R_{E}}$ mice, and wild-type controls. Local inducible precursors expressing BMP receptors interact with the BMP2 in the sponges and inducible precursors in skin will develop into bone. Thus, the collagen scaffold with BMP2 will result in formation of a bone/marrow organ with outer cortical and inner trabecular bone interspersed with marrow cells and adipocytes. At 4 weeks post transplantation, BMP2-induced-ossicles were harvested for microCT scans and histology analysis. Ossicles formed in Tg6 mice (Fig. 6a) had reduced trabecular and cortical BV (Fig. 6e, f) compared to those formed in littermate control. Histology analysis (Fig. 6c) revealed extensive erythropoiesis, less bone, fewer adipocytes (Fig. 6g), and increased marrow (Fig. 6h) in Tg6-ossicles. Features in ossicles formed in Tg6 mice were consistent with reduced bone and marrow adipocytes, and hypercellular marrow observed in these mice (Figs. 1 and 3a). Analysis of TRAP stained sections of ossicles showed fewer osteoclasts in Tg6-ossicles (Fig. 6i), suggesting that reduced bone in Tg6-ossicles is not due to resorption by osteoclasts. This is in contrast to the increased osteoclast number and bone reduction observed in Tg6 femur sections (Fig. 5a, e). Ossicles formed in $\triangle$ EpoR $_{E}$ mice (Fig. 6b) also had lower trabecular and cortical BV (Fig. 6j, k) compared with ossicles that developed in control mice. These features were consistent with the reduced bone phenotype observed in $\triangle E p o R_{E}$ mice (Fig. 2). Histology analysis (Fig. 6d) of $\Delta$ EpoR $_{\mathrm{E}}$-ossicles did not show any significant differences in adipocytes, marrow, and in the number of osteoclasts found on bone surface (Fig. 6l-n).

Elevated EPO signaling in BMSCs reduces their differentiation into osteoblasts and adipocytes

We performed colony formation efficiency assays with bone marrow cultures of wild-type, Tg6-, and $\Delta$ EpoR $_{\mathrm{E}}$-mice $(n=5-6 /$ group) to assess the colony forming potential of BMSCs present in the bone marrow. BMSCs from Tg6 mice showed reduced colony forming ability (Supplementary Fig. 5a), whereas we did not observe any defect in the colony forming efficiency in the BMSCs from the $\triangle E p o R_{E}$ mice (Supplementary Fig. 5b). Since in vitro culture conditions lack the extensive regulatory systems present in vivo, to further investigate EPO regulation of BMSC differentiation into osteoblasts or adipocytes we performed in vivo ectopic bone formation studies following BMSC transplantation into immunodeficient NSG mice.

Isolation of BMSCs by culturing adherent cells of bone marrow followed by depletion of $\mathrm{CD} 45^{+} \mathrm{CD} 11 \mathrm{~b}^{+}$cells by magnetic sorting to exclude all hematopoietic cells including contaminating macrophages has been shown to yield pure BMSCs that can be used for in vivo bone formation assays. ${ }^{29}$ These cells express positive BMSC markers, which are stable for cell passages until $8-10$. We used these validated protocols to isolate BMSCs from wild-type, Tg6-, and $\Delta$ EpoR $_{E}$-mice ( $n=4-5 /$ group), to perform in vivo bone formation assays. ${ }^{30}$ BMSCs from Tg6 mice express human EPO, and also express Epor in comparable levels to that of wild-type littermates (Supplementary Fig. 4e, f). $\triangle$ Epo $_{\mathrm{E}}-\mathrm{BMSCs}$ do not express Epor (Supplementary Fig. 4g). Isolated BMSCs were absorbed into a collagen scaffold and surgically transplanted subcutaneously into immunodeficient mice. Each mouse received one each of wild-type and Tg6- or $\Delta E \mathrm{EpoR}_{\mathrm{E}}$-mice transplants, as well as one collagen sponge without any cells. In this assay, BMSCs will differentiate and form a bone/marrow organ (Fig. 7a-d) consisting of bone, adipocytes, and stroma of donor origin, whereas the hematopoiesis originates from the recipient mice. ${ }^{31}$ BMSCs from each mouse were transplanted into a minimum of three different recipient mice to obtain consistent results. MicroCT analysis of ossicles after 8 weeks post transplantation showed reduced osteogenic potential of Tg6-BMSCs compared to wildtype (Fig. 7a). Histology analysis showed that Tg6-BMSCs ossicles did not develop into well-defined cortical and trabecular bone structures as seen in ossicles formed from wild-type BMSCs (Fig. 7c). There were hardly any trabecular features in most of the ossicles formed by Tg6-BMSCs and, for this reason, trabecular parameters could not be calculated for Tg6-BMSCs (Fig. 7e). Analysis of cortical bone formation showed Tg6-ossicles having less cortical BV (Fig. 7f). Tg6-BMSCs also had reduced adipogenesis and marrow formation (Fig. 7c, g, h). There was no change in hematocrit levels of recipient mice transplanted with Tg6-BMSCs (data not shown). There were also no significant differences in number of osteoclasts in Tg6-ossicles (Fig. 7i). Ossicles formed by $\triangle \mathrm{EpoR}_{\mathrm{E}}-\mathrm{BMSC}$ had a similar structure to that of control (Fig. 7b, d); however, separation of cortical and trabecular bone suggested ossicles formed by $\triangle$ EpoR $_{E}-B M S C s$ had a trend for fewer trabeculae (Fig. 7j) with no change in cortical BV (Fig. 7). $\triangle E_{p o R_{E}}$ mice had enhanced adipogenic differentiation capacity and enhanced marrow (Fig. 7l, m). Analysis of osteoclasts showed no differences in ossicles formed by $\triangle E$ po $R_{E}-B M S C s$ when compared with wild-type controls (Fig. 7n).

Gene expression analysis of Tg6-BMSCs used for ectopic bone formation assay showed reduced Ppar- $\gamma$ and Osterix expression, which is consistent with reduction in both adipogenesis and osteogenesis (Suppl Fig. 4h). Tg6-BMSCs also expressed more Gata2 and Gata3. Increased Gata2 and Gata3 have been reported to inhibit 
a

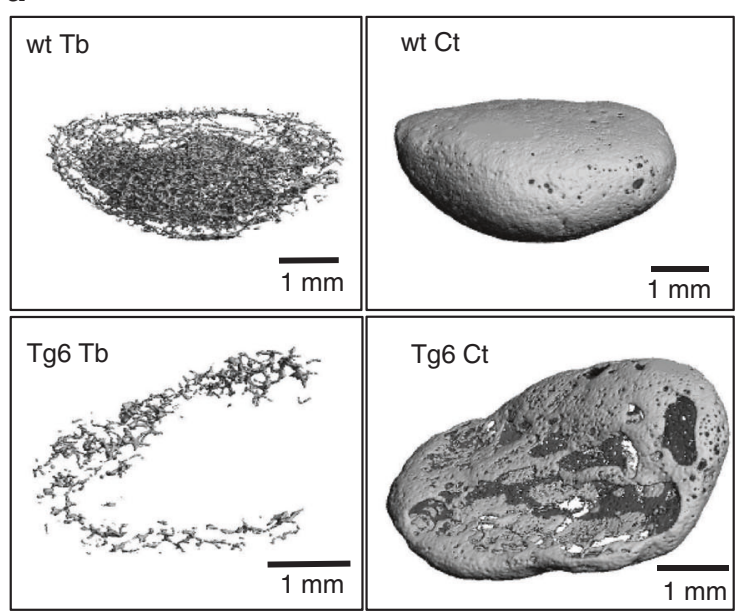

c

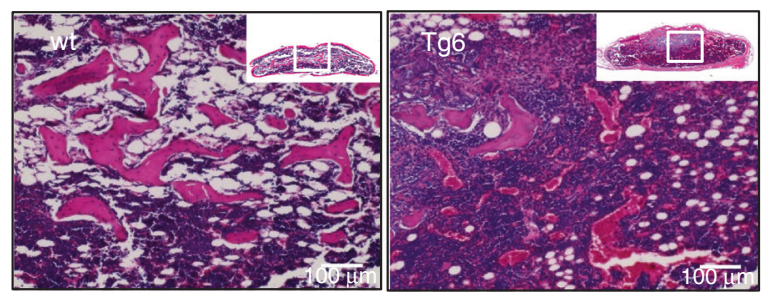

b

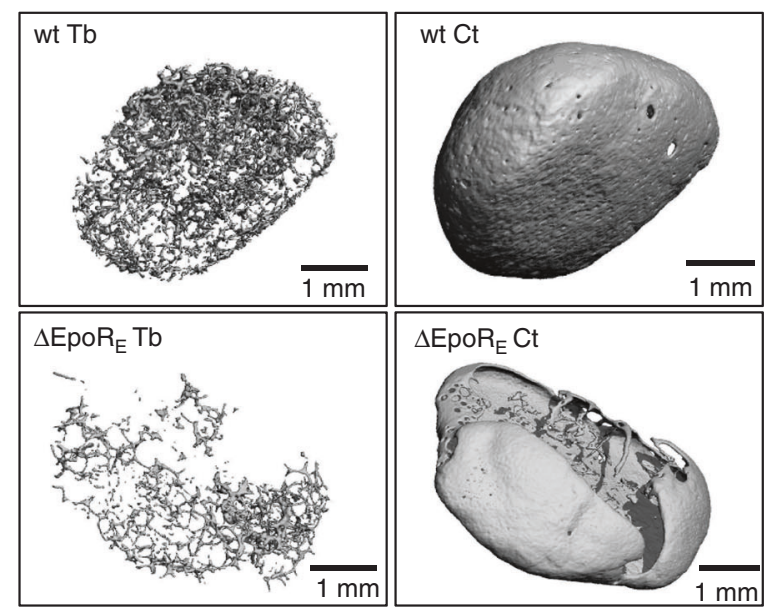

d

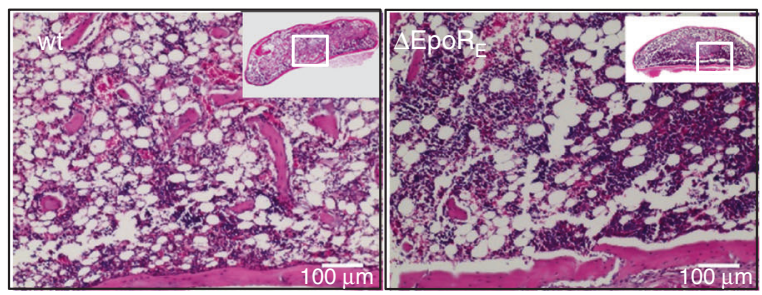

e

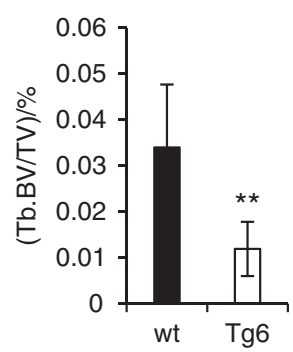

f
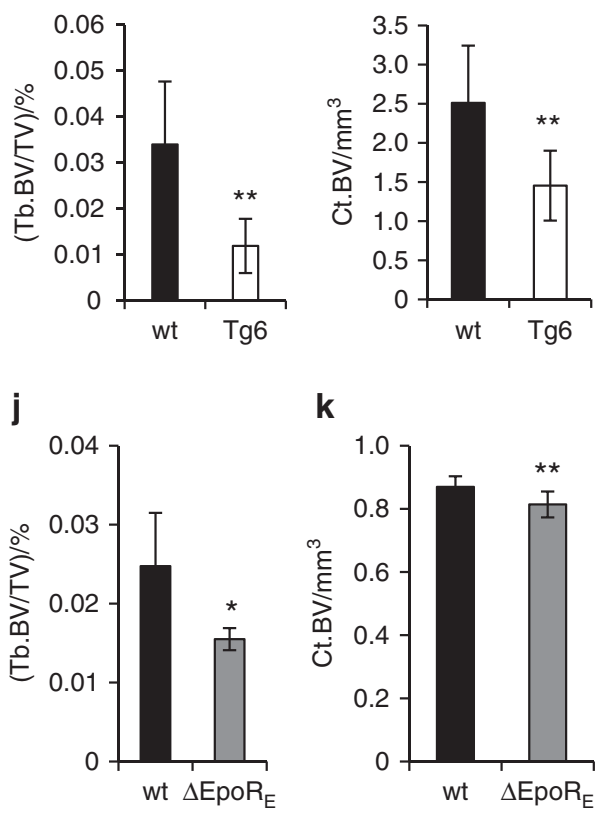

k

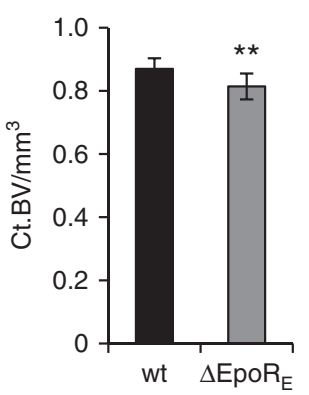

g
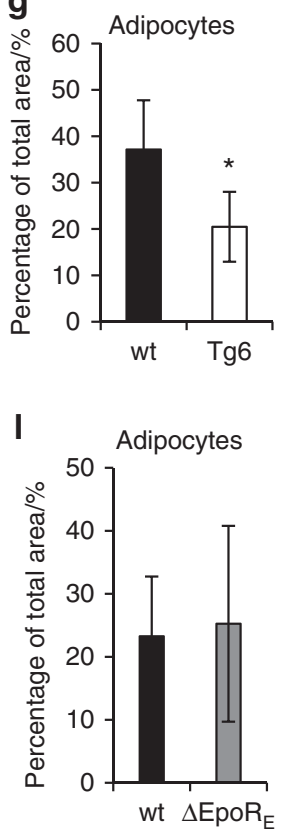

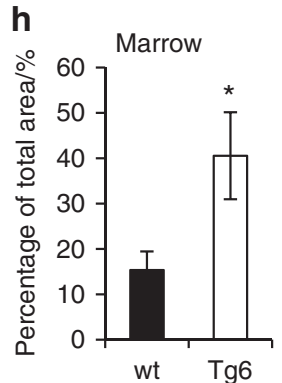

i

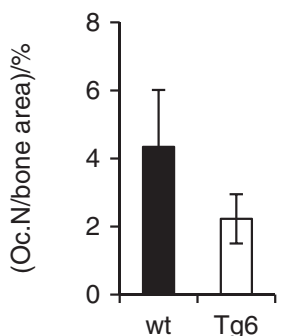

Fig. 6 Elevated EPO and lack of EPOR signaling inhibit BMP2-induced ectopic bone formation. a Micro-CT images of ectopic bone formed by BMP2 in collagen scaffolds transplanted in wild-type (wt) and Tg6-mice 4 weeks post transplantation. Shown are ossicles formed in wt mice (top) and in Tg6-mice (bottom), and inner trabecular bone (right) and cortical bone (left). b Micro-CT images of ectopic bone formed by BMP2 in collagen scaffolds transplanted in wild-type (wt) and $\Delta \mathrm{EpoR}_{\mathrm{E}}$-mice 4 weeks post transplantation. Shown are ossicles formed in wt mice (top) and in $\Delta$ EpoR $_{\mathrm{E}}$-mice (bottom), and inner trabecular bone (right) and cortical bone (left). c H\&E staining of decalcified ossicles formed in the wt and Tg6-mice, showing bone as pink colored regions, adipocytes as white circular regions and marrow as blue regions. $\mathbf{d} \mathrm{H} \& \mathrm{E}$ stained images of ossicles formed in wt and $\Delta$ EpoR $_{\mathrm{E}}$-mice bone, marrow and adipocytes. e, $\mathbf{f}$ Micro-CT quantification of trabecular (e) and cortical (f) volume of the ectopic bone formed in wt and Tg6-mice. g Quantification of marrow adipocytes in the wt and Tg6-ossicle sections. $\mathbf{h}$ Space occupied by marrow (excluding adipocytes and bones). i Number of TRAP stained osteoclasts in the wt and Tg6-ossicles. $\mathbf{j}$, $\mathbf{k}$ Micro-CT quantification of trabecular (j) and cortical (k) volume of the BMP2-induced ectopic bone formed in wt and $\Delta$ EpoR $_{\mathrm{E}}$-mice. I Quantification of marrow adipocytes in the wt and $\Delta$ EpoR $_{\mathrm{E}}$-Ossicle sections. $\mathbf{m}$ Space occupied by marrow (excluding adipocytes and bone). $\mathbf{n}$ Number of TRAP stained osteoclasts in the wt and $\Delta$ EpoR $\mathrm{R}_{\mathrm{E}}$-ossicles. Quantification of adipocytes, marrow space and osteoclast numbers were performed using ImageJ analysis. $\left(n=4-5 /\right.$ group, $\left.{ }^{*} P<0.05,{ }^{* *} P<0.01\right)$ 


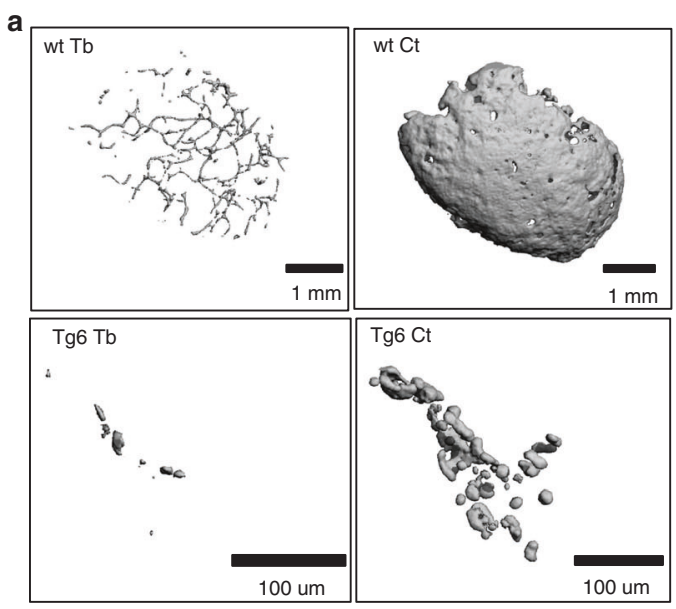

b
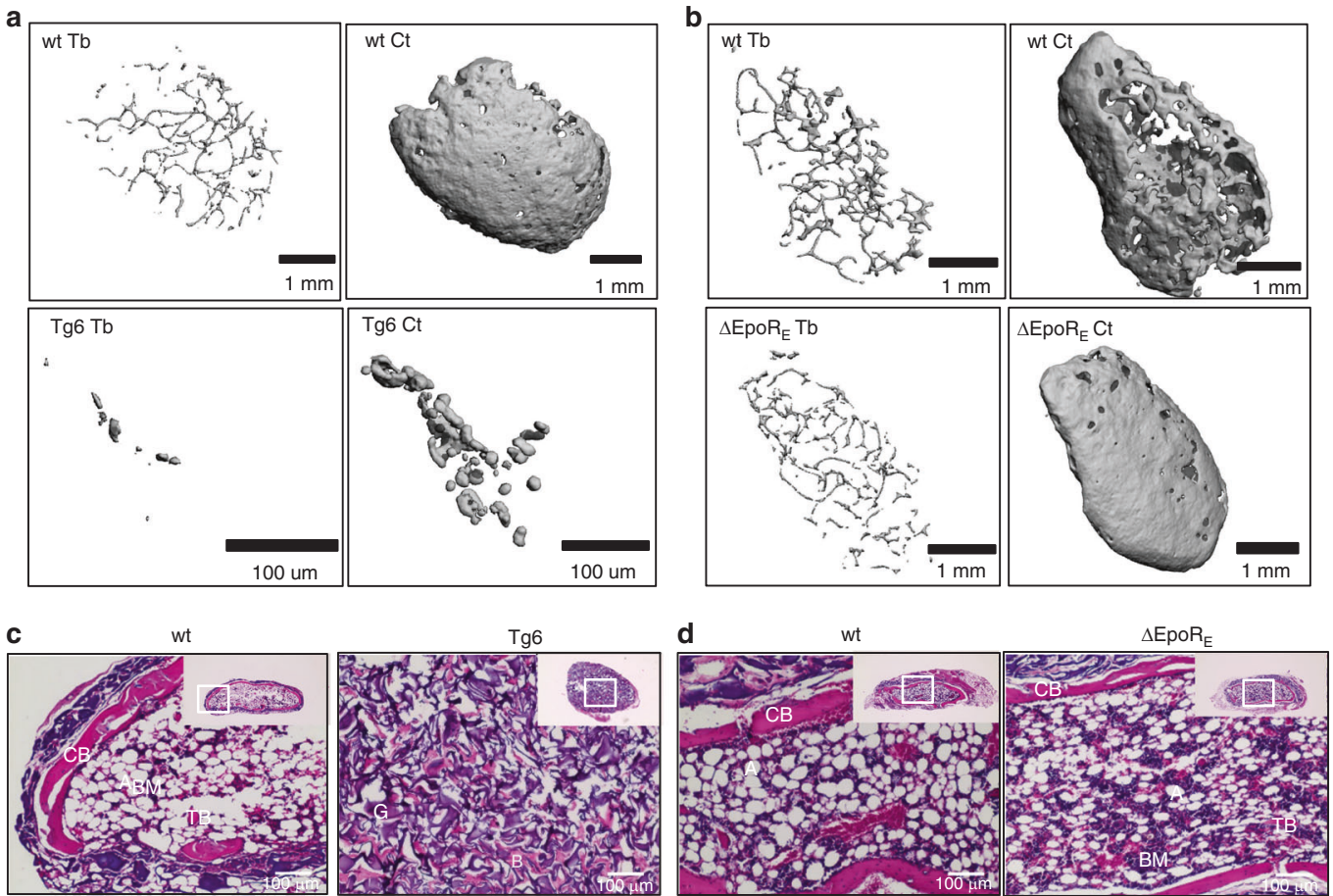

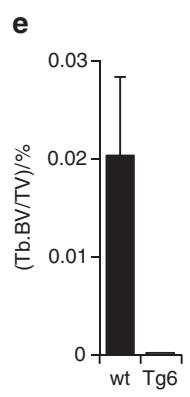

j

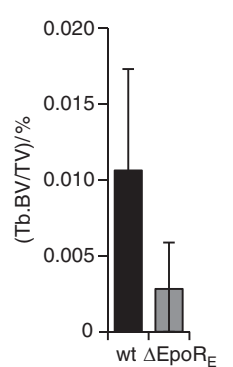

f

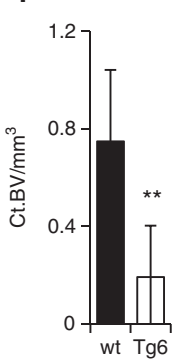

$\mathbf{k}$

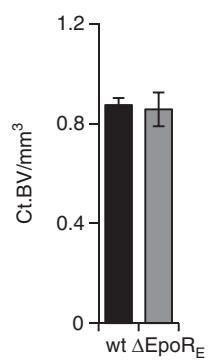

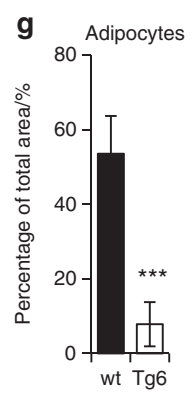

I

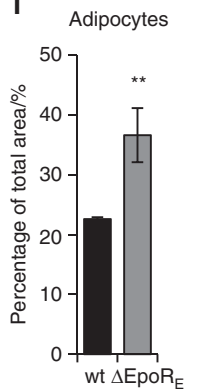

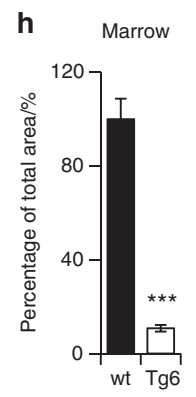

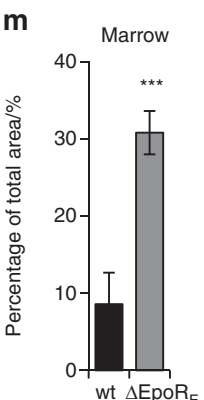

i

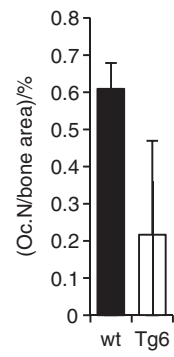

n

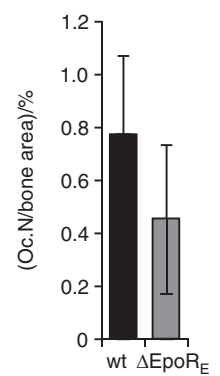

Fig. 7 In vivo bone formation potential of BMSCs from Tg6- and $\triangle E_{\text {EpoR }}$-mice transplanted in immunocompromised mice. a Micro-CT images of ectopic bone formed by the differentiation of BMSCs from wild-type (wt) and Tg6-mice 4 weeks post transplantation. Shown are wt BMSC ossicles (top) and Tg6-BMSC ossicles (bottom), and inner trabecular bone (right) and cortical bone (left). b Micro-CT images of ectopic bone formed by BMSCs from wild-type (wt) and $\triangle E_{\mathrm{EpoR}}$-mice. Shown are wt BMSC ossicles (top) and $\triangle \mathrm{EpoR}_{\mathrm{E}}-\mathrm{BMSC}$ ossicles (bottom), and inner trabecular bone (right) and cortical bone (left). c, d H\&E staining of wt, Tg6 (c), and $\triangle E p o R_{E}$ (d) BMSC ossicles showing cortical bone (CB), trabecular bone (TB), adipocytes (A), gelfoam (G), and bone marrow (BM). e, $\mathbf{f}$ Quantification of trabecular (e) and cortical bone volume (f) of wt and Tg6-BMSC ossicles. $\mathbf{g}$, $\mathbf{h}$ Area of adipocytes (g) and bone marrow (h) in wt and Tg6-BMSC ossicles quantified using ImageJ software analysis. $\mathbf{i}$ Quantification of the number of osteoclasts present in the ossicles developed from the wt and Tg6-BMSCs. $\mathbf{j}$, $\mathbf{k}$ Micro-CT quantification of trabecular (j) and cortical bone volume $(\mathbf{k})$ of ossicles developed from the differentiation of wt and $\Delta$ EpoR $\mathrm{R}_{\mathrm{E}}-\mathrm{BMSCs}$. I, $\mathbf{m}$ Area of adipocytes (I) and bone marrow $(\mathbf{m})$ inside the wt and $\Delta$ EpoR $_{\mathrm{E}}$-ossicles. $\mathbf{n}$ Quantification of osteoclasts present on the wt and $\Delta \mathrm{EpoR}_{\mathrm{E}}-\mathrm{BMSC}$ ossicles. $\left(n=4\right.$ /group, $\left.{ }^{* *} P<0.01,{ }^{* * *} P<0.001\right)$

Ppar- $\mathrm{Y}$ and suppress adipogenesis. ${ }^{32} \Delta$ EpoR $_{\mathrm{E}}-\mathrm{BMSC}$ had more PparY expression and reduced Gata3 levels (Supplementary Fig. 4i), which is consistent with their increased adipogenesis. Unlike Tg6-BMSCs with reduced Osterix expression, in $\triangle$ EpoR $_{\mathrm{E}}-\mathrm{BMSC}$ Osterix expression was elevated and ossicle formation was similar to wild-type BMSC. We assessed the expression of Epor in BMSCs relative to other cells and tissues that express Epor. Even though, the Epor levels are low in BMSCs, our data show intrinsic EPO signaling as an essential factor for their differentiation (Supplementary Fig. 5c). Therefore, endogenous EPO is required to maintain BMSC balance 
a Wild type BMSC differentiation

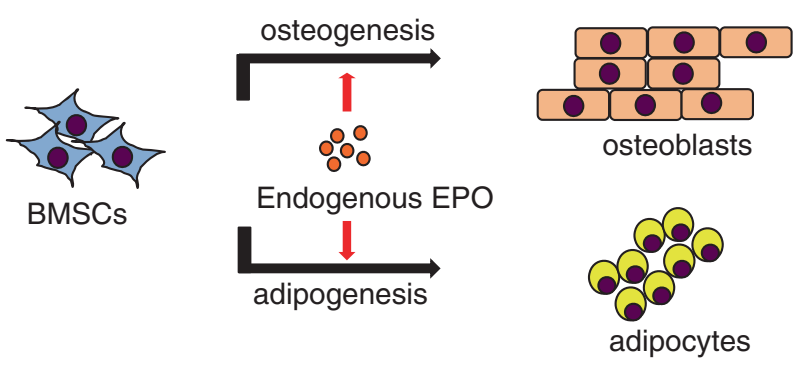

b Tg6 BMSC differentiation
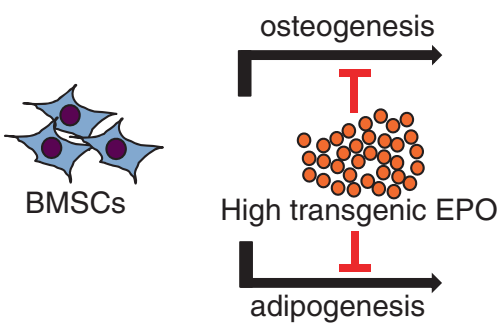

$\underset{\text { osteoblasts }}{0} 0$

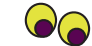

adipocytes

d EPO treatment and BMSC differentiation

C $\triangle \mathrm{EpoR}_{\mathrm{E}}$ BMSC differentiation

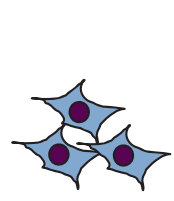

BMSCs

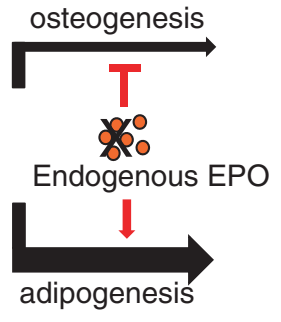

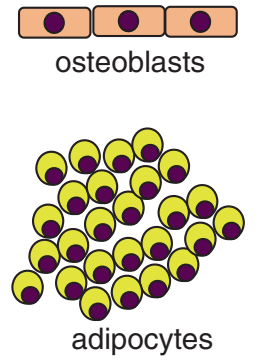

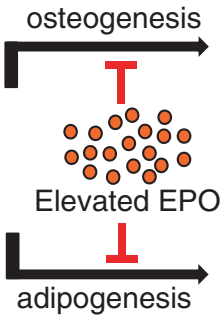

adipogenesis

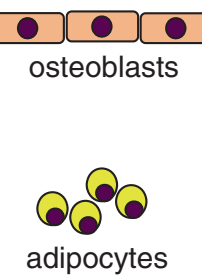

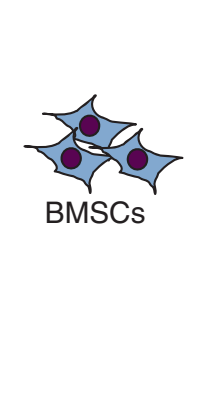

Fig. 8 Schematic for erythropoietin regulation of BMSC differentiation. a EPO-EPOR signaling in BMSCs is required for maintaining the balance between osteogenic and adipogenic differentiation in the marrow. b Elevated EPO signaling in Tg6-mice inhibits both osteogenesis and adipogenesis. c Absence of endogenous EPO signaling in BMSCs in $\triangle$ EpoR $_{\mathrm{E}}$-mice reduces osteogenesis and favors adipogenesis resulting in fatty marrow. d EPO treatment in mice inhibits both osteogenesis and adipogenesis

between osteogenesis and adipogenesis while high EPO inhibits both osteogenic and adipogenic differentiation (Fig. 8).

\section{DISCUSSION}

In this study, we addressed the role of EPO signaling in bone, BMSCs and bone marrow adipocytes in Tg6 mice with chronic elevated EPO, wild-type mice with acute EPO treatment, and $\triangle$ EpoR $_{E}$ mice lacking EPO signaling in non-erythroid cells. We recently observed a sex-differential role of EPO in metabolic regulation where elevated EPO reduced external fat depots in male mice but not in female mice. ${ }^{33}$ Here, we find that reduction in marrow fat with elevated EPO is not gender-specific as both male and female Tg6-mice had similar reduction in marrow fat. However, we noted gender differences might contribute to some of the variations in trabecular parameters we observed in male Tg6 mice compared with previous report for female Tg6 mice. ${ }^{22}$ In our study, male Tg6 mice had extensive reduction of trabecular bone with decreased trabecular thickness, whereas Tg6 female mice had increased trabecular thickness. Only male Tg6-mice had reduced femoral length and body weight, whereas female Tg6mice did not differ from their littermates in these parameters.

We report that endogenous EPO is important for bone development and marrow adipogenesis as $\Delta E p o R_{E}$ mice had reduced trabecular bone and increased marrow adipocytes, and these features amplified with age. In mice, age related changes in bone microarchitecture occur early in life with peak trabecular BV between the ages of 6 and 8 weeks followed by a steady decline specifically in the long bone metaphyses. ${ }^{34}$ In our study, in the younger $\triangle E$ poR $R_{E}$ mice of 8 weeks of age, the absence of endogenous EPO signaling showed early signs of reduction in trabecular bone with fewer trabeculae and increased spacing. However, at 11 weeks of age, reduction in trabecular bone becomes apparent with significant reduction in trabecular BMD and trabecular BV. The $\triangle$ EpoR $_{\mathrm{E}}$-mice gains body weight and fat mass with age because of lack of Epo signaling in non- hematopoietic tissues. ${ }^{8}$ Increased fat mass is associated with lower BMD in mice ${ }^{35}$ and in humans obesity is reported to show low bone formation. ${ }^{36}$ Compared to body weight matched wildtype mice, the $\Delta \mathrm{EpoR}_{\mathrm{E}}$-mice had reduced trabecular bone ruling out the role of body weight as a factor for reduced bone phenotype. But, we cannot exclude the possibility of increased fat mass in the 11-week-old $\triangle$ Epo $_{\mathrm{E}}$ mice contributing to a low bone phenotype in our study.

Endogenous EPO is also important in osteoclast differentiation particularly as mice become older as we observed increased osteoclasts in the 11-week-old $\Delta \mathrm{EpoR}_{\mathrm{E}}$ mice. These mice also have systemic inflammation ${ }^{25}$ and gain body weight with age. Therefore, the increase in osteoclasts seen in 11-week-old mice might be associated with their high level of inflammation consistent with previous reports linking increased osteoclast differentiation to inflammation. ${ }^{37}$ In mice models, induction of systemic inflammation by methods like high-fat diet feeding ${ }^{35}$ or administration of lipopolysaccharide is associated with enhanced osteoclastogenesis causing bone resorption resulting in bone loss. ${ }^{38}$ Thus, the reduction in bone seen in the 11-week-old $\Delta$ Epo $_{E}$ mice could also be a consequence of inflammation arising due to the lack of Epo signaling in non-hematopoietic tissues. Therefore, we assessed bone parameters in younger $\Delta \mathrm{EpoR}_{\mathrm{E}}$ mice 8 weeks of age where their body weight is similar to control mice. In young $\triangle E p o R_{E}$ mice we still observed reduction in trabecular bone, but there was no increase in osteoclasts or increased Cathepsin $K$ suggesting the initial disruption of trabecular bone occurring in young $\triangle \mathrm{EpoR}_{\mathrm{E}}$ mice as an osteoclast-independent mechanism. Thus our data show endogenous EPO as an essential cytokine with varying levels of regulation in the bone environment. In younger mice with rapid bone development as well as in older mice with age induced skeletal disruption endogenous EPO is required for normal bone development particularly in the maintenance of trabecular bone microarchitecture.

Exogenous EPO administration increases hematocrit in a dose dependent manner ${ }^{39}$ and in mice both low dose $\left(300 \mathrm{U} \cdot \mathrm{kg}^{-1}\right)^{19}$ 
and high dose $\left(9000 \mathrm{U} \cdot \mathrm{kg}^{-1}\right)^{22}$ of EPO administration reduced trabecular bone in long bones. However, whether EPO-induced bone loss is associated with EPO stimulated erythropoiesis or is entirely mediated by non-erythroid cells is not known. Therefore, we injected young wild-type and $\triangle E p o R_{E}$ mice 6 weeks of age with $1200 \mathrm{U} \cdot \mathrm{kg}^{-1}$ rhEPO for 10 days to achieve increased hematocrit during the period of maximum bone remodeling in mice. Unlike wild-type mice, EPO treatment in $\triangle E p R_{E}$ mice did not result in trabecular bone loss despite increasing hematocrit levels comparable to EPO treated wild-type mice. This provides evidence that reduced bone parameters seen with elevated EPO is independent of erythropoiesis and is mediated by non-erythroid cell response.

The role of endogenous EPO signaling in marrow adipogenesis has not been reported. The increased marrow adiposity in $\triangle E p o R_{E}$ mice shows that lack of EPO signaling in non-erythroid cells leads to a fatty marrow. The $\triangle E p o R_{E}$ mice gain increased body weight because of accumulation of white fat mass and this accrual increases with age. ${ }^{8}$ In younger $\Delta \mathrm{EpoR}_{\mathrm{E}}$ mice of 8 weeks of age, we observed significant increase in marrow adipocytes even before these mice started to gain weight compared to the age-matched wild-type mice. These data show endogenous EPO signaling as an important regulator of marrow adiposity. In the bone marrow of female mice there was extensive adiposity compared with agematched male mice. A steep increase in marrow adiposity is also observed in menopausal women with $10 \%$ more vertebral marrow fat than men of similar age. ${ }^{40}$ The reduction in marrow adipocytes in both wild-type and $\triangle \mathrm{EpoR}_{\mathrm{E}}$-mice with EPO administration shows that this process is due to EPO stimulated erythropoiesis in the marrow. Thus, EPO regulation of bone marrow adipocytes is different from EPO regulation of adipocytes in external fat depots. As previously reported, $\triangle E_{p o R_{E}}$ mice that do not express Epor in adipose tissue do not lose white fat mass with EPO treatment despite increased hematocrit. ${ }^{8}$ Loss of bone marrow adipocytes with EPO treatment does not depend on the presence of Epor on these cells as both wild-type and $\triangle E_{p o O} R_{E}$-mice lose bone marrow adipocytes. However, endogenous EPO-EPOR signaling is important for regulated bone marrow adipogenesis because its absence results in fatty marrow in $\triangle E p o R_{E}$ mice. These data suggest that the reduction in bone and marrow fat by elevated EPO is mediated differently. With bone loss, we observe an erythropoietic independent action of EPO, whereas reduction in marrow adipocytes is associated with EPO stimulated increased erythroid cell production in the marrow.

The reduced bone observed in Tg6 mice and in wild-type mice receiving EPO has been associated with increased osteoclasts. ${ }^{22}$ Our findings in Tg6-mice were similar to the previous report, but we did not observe osteoclast induction in mice with acute EPO treatment despite bone loss. Bone loss in wild-type mice treated with EPO without an increase in osteoclasts was also previously observed. ${ }^{41}$ Similarly, addition of EPO did not stimulate osteoclast differentiation in vitro, consistent with report on lack of EPO effect in in vitro osteoclast cultures. ${ }^{19}$ Thus, elevated EPO in chronic and acute conditions reduce trabecular bone possibly by different mechanisms. Increase in osteoclasts with chronic elevated EPO can contribute to bone reduction, but is less evident in acute EPO treatment. The response of osteoclasts to chronic EPO is known and we also observed increased osteoclasts in the Tg6-mice bones and marrow cultures, but the osteoblast response to chronic EPO in the Tg6-mice has not been previously reported. We observed increased Alp, Osterix expression in the primary osteogenic cells from these mice along with increased mineralization in cultures. Early reports of analysis of bone markers in Tg6 mice identified increased serum TRAP and reduced serum Osteocalcin levels, ${ }^{22}$ suggesting a high bone resorption and low bone formation rate. Based on gene expression and in vitro osteogenic cultures from our studies along with information on the bone markers in Tg6 mice, it can be concluded that chronic exposure to high levels of
EPO results in both increased osteoclast and osteoblast activity causing high bone turnover resulting in bone reduction.

We find elevated EPO reducing Bmp levels in both chronic and acute conditions. Bmp2 and Bmp6 are important osteogenic factors, ${ }^{42}$ produced in the bone marrow by macrophages, osteoblasts, and chondrocytes. ${ }^{43-45}$ Expression of EPOR by all these cells $s^{20,46}$ raises the potential for regulation of BMP signaling by EPO. In Tg6 mice, high EPO levels inhibited the ectopic bone formation potential of BMP2, in an osteoclast independent manner. In this assay, ossicles formed in Tg6 mice were exposed to elevated EPO only for 4 weeks, a duration similar to acute EPO treatment in mice. In both these conditions, increased EPO did not increase osteoclasts but reduced bone suggesting that the primary response of elevated EPO in bone is not to stimulate bone resorption but to inhibit bone formation. Elevated EPO exposure for a prolonged period of time might eventually increase osteoclasts and contribute to reduced bone as seen in Tg6 mice. Previous studies showed EPO synergistically interacting with BMP2 to promote bone formation ${ }^{47,48}$ in calvarial defect models where EPO promotes tissue repair by augmenting BMP2 induced osteogenesis. In addition to reducing bone formation, we observed that elevated EPO increased marrow and reduced adipocytes in ectopic bone, consistent with an earlier report. ${ }^{48}$ In the $\triangle E_{p o R_{E}}$ mice, we observed reduced $B m p 2$ in the marrow and a reduction in BMP2-induced ectopic bone formation. Therefore, both elevated EPO and disrupted endogenous EPO signaling inhibits BMP2-induced osteogenesis and aberrant BMP signaling could be one mechanism by which osteogenesis is impaired under these conditions.

Precursors of osteoblasts in the postnatal organism are BMSCs, and we examined the function of EPO signaling on BMSC differentiation in conditions of elevated EPO and in the absence of endogenous EPO signaling using in vivo bone formation assays. Tg6-BMSCs with increased EPO signaling had limited differentiation ability for both osteoblast and adipocyte lineages. These cells have increased Gata2 and Gata3 expression in the Tg6-BMSCs with reduced Ppar-y expression. GATA2 and GATA3 have been shown to inhibit PPAR- $\gamma$ activity and thereby reduce adipogenesis. ${ }^{49}$ The essential role of endogenous EPO signaling in BMSC differentiation is evident from the wild-type BMSCs expressing Epor, which formed ectopic bone with distinct trabecular and cortical bone with well distributed adipocytes in the marrow space. Conversely, BMSCs from $\triangle E_{p o R} R_{E}$ mice, which do not express Epor, and therefore do not respond to circulating EPO in the recipient mouse, formed bone with less trabeculae and more adipocytes. Report of osteoblast production of EPO directly modulates erythropoiesis raising the possibility of EPO/EPOR autocrine response even with low Epor expression in osteoblasts. ${ }^{50}$ Furthermore, a direct effect of EPO in cultures of primary mouse BMSCs was indicated by treatment with low dose EPO that inhibited expression of osteoblast specific genes, Alp, Runx2, and Osteocalcin while siRNA silencing of Epor completely abrogated the reduction of osteoblast markers. ${ }^{41}$ In cultures of human BMSCs, EPO treatment increased cell viability, ALP activity, and cell mineralization dependent on JAK2, PI3K, and mTOR pathways, which was inhibited by mTOR inhibitor Rapamycin. ${ }^{51}$ Similarly, Rampamycin also was reported to inhibit EPO induced osteogenic differentiation of human BMSC cultures. ${ }^{52}$

Increased Ppar- $\gamma$ expression in $\triangle$ Epo $_{\mathrm{E}}-\mathrm{BMSC}$ s is consistent with their increased adipogenic differentiation potential. In vitro differentiation studies using BMSCs have shown EPO promoting their differentiation to osteoblasts. ${ }^{52}$ We performed in vitro differentiation assays with whole bone marrow cultures, but had frequent macrophage overgrowth during the long-term culture conditions required for differentiation assays. Differentiation assays with sorted primary BMSCs also were not possible as the BMSCs did not survive by themselves after couple of days in culture. Our data show that the EPO effect in BMSC differentiation 
in vivo is different from in vitro culture conditions reported previously. The ectopic bone/marrow formation assay with BMSCs also reflects the bone architecture of the mouse models we used; Tg6-BMSCs have decreased osteogenesis and adipogenesis and Tg6 mice have reduced bone and marrow adipocytes (Figs. 1 and 3). $\triangle E p o R_{E}-B M S C s$ developed less trabecular bone and more bone marrow adipocytes consistent with the bone in these mice (Figs. 2 and 3). Thus, absence of EPO signaling disproportionately increases the differentiation of BMSCs to marrow adipocytes with reduction in osteogenesis, while high EPO signaling in BMSCs decreases their differentiation to either lineages.

In conclusion, we provide evidence for the essential role for both endogenous EPO and elevated EPO signaling in the development of bone and marrow adipocytes. Disrupted differentiation of BMSCs due to altered cytokine signals in the marrow environment enhances adipogenesis while diminishing the osteogenic potential of BMSCs. ${ }^{53-55}$ Bone marrow adipocytes have a distinct origin and function compared with adipocytes comprising white and brown adipose tissue. ${ }^{56,57}$ With recent improvements in the measurement of bone marrow adipocytes in humans ${ }^{58}$ and in animal models, ${ }^{59}$ studies addressing the role of EPO signaling in the bone marrow fat compartment will shed new insights in understanding the non-hematopoietic roles of EPO in development and disease.

\section{METHODS}

Mice models

Tg6 mice overexpressing PDGF $\beta$ promoter driven human $\mathrm{EPO}^{23}$ and $\triangle \mathrm{EpoR}_{\mathrm{E}}$ mice that are Epor-/- mice rescued by erythroid restricted Epor transgene (GATA-1 locus hematopoietic regulatory domain driving mouse Epor CDNA), ${ }^{24}$ established on C57BL6/J background, were used as models for chronically elevated EPO and lack of EPOR signaling in non-erythroid cells, respectively. Wildtype mice (C57BL6/J) were obtained from (Jackson Laboratory, ME, USA). Recombinant human epoetin-a (Amgen, CA, USA) was administered to wild-type and $\triangle E p o R_{E}$-mice subcutaneously at a dose of $1200 \mathrm{IU} \cdot \mathrm{kg}^{-1}$ daily for 10 days. Control mice were injected with phosphate buffered saline (PBS) (Gibco, Life Technologies, USA). For SSC/BMSC transplantation, 8-week-old female immunocompromised NSG mice (NOD.Cg-Prkdcscidll2rgtm1Wjl/Szj; Jackson Laboratory, ME, USA) were used. All animal procedures were conducted under the National Institutes of Health guidelines.

\section{Micro-CT analysis}

Mouse femurs were analyzed using a Bruker Skyscan 1172 microCT scanner (Micro Photonics Inc, PA, USA). Trabecular and cortical regions were analyzed at $0.35 \mathrm{~mm}$ and $4.25 \mathrm{~mm}$ from the growth plate, respectively. For both regions, $1.5 \mathrm{~mm}$ of femur bone sections comprising 257 bone slices were individually analyzed using CTAn software at $6 \mu \mathrm{m}$ resolution and 3D models of regions of interest were constructed. The regions of interest were similar in 8-week and 11-week-old mice analyzed in this study.

\section{Bone histology}

Mouse femurs were fixed in $10 \%$ buffered neutral formalin (Sigma, MO, USA) for $24 \mathrm{~h}$ followed by decalcification in $10 \%$ EDTA $\left(0.5 \mathrm{~mol} \cdot \mathrm{L}^{-1}\right)$ at $4{ }^{\circ} \mathrm{C}$ until complete decalcification was confirmed by $x$-ray analysis. Hematoxylin-Eosin (H\&E) staining was performed on $6 \mu \mathrm{m}$ sections of decalcified paraffin embedded femurs to assess bone marrow histology. For osteoclast detection in femurs, Tartrate-resistant acid phosphatase (TRAP) staining was performed using TRAP/ALP Stain Kit according to the manufacturer's instructions (\#294-67001, Wako Chemicals, USA).

\section{Osteoclast generation from bone marrow}

Bone marrow cells were harvested from 8- to 10-week-old mice and seeded into tissue culture treated plates and allowed to attach overnight in a-MEM medium with $10 \%$ FBS. Nonadherent cells $\left(5 \times 10^{5}\right.$ cells $\left./ \mathrm{cm}^{2}\right)$ were plated into 96 flat-bottomed well plates in fresh medium containing $30 \mathrm{ng} \cdot \mathrm{mL}^{-1}$ RANKL and $20 \mathrm{ng} \cdot \mathrm{mL}^{-1} \mathrm{M}$-CSF (Peprotech, NJ, USA). Medium was replaced every other day until osteoclasts appeared (4-5 days). TRAP staining was performed according to manufacturer's instructions (\#387-1A, Sigma, St Louis, MO, USA) to visualize osteoclasts.

\section{Osteogenic cultures from calvaria}

Calvarial osteogenic cells were isolated from 6 to 8 weeks old mice using previously described protocol. ${ }^{60}$ Briefly, calvarial pieces without red hematopoietic regions were placed in solution of collagenase II and dispase ( $4 \mathrm{mg} \cdot \mathrm{mL}^{-1}$ each) in PBS (Worthington Biochemical Corp, NJ, USA). Calvarial pieces were incubated for $5 \times 15$ min changes total of collagenase II and dispase solution on a shaker at $37^{\circ} \mathrm{C}$. Cells obtained from the first 15 min incubation were discarded to exclude hematopoietic cells. Cells from the remaining four changes were pooled in tubes of fresh a-MEM medium with $20 \% \mathrm{FBS}$, centrifuged and plated in a-MEM medium with $20 \% \mathrm{FBS}$ in $25 \mathrm{~cm}^{2}$ tissue culture flask. Fresh medium was replaced the next day followed by medium change twice weekly. Once cells reach confluence (1-2 weeks), they were incubated in medium containing $5 \mathrm{mmol} \cdot \mathrm{L}^{-1} \quad \beta$-glycerophosphate and $100 \mu \mathrm{g} \cdot \mathrm{mL}^{-1}$ ascorbic acid (Sigma, MO, USA) with medium replacement twice weekly.

Viability, differentiation and mineralization assays

Viability of osteogenic cells was measured using Thiazolyl Blue Tetrazolium Blue (MTT) assay (Sigma). Alkaline phosphatase (ALP) activity was quantified using SIGMAFAST ${ }^{\mathrm{TM}}$ p-Nitrophenyl phosphate Tablets (Sigma). For microscopic observation of ALP activity, SIGMA FAST ${ }^{T M}$ BCIP/NBT (5-Bromo-4-chloro-3-indolyl phosphate/ Nitro blue tetrazolium) tablets were used according to manufacturer's instructions. Mineralization was detected using 1\% alizarin staining ( $\mathrm{pH}$ 4.6). All reagents for measuring ALP activity, ALP visualization and mineralization were purchased from Sigma, MO, USA.

\section{Colony forming unit assay}

Estimation of SSCs in the BMSC population was determined by colony forming efficiency assay as described previously. ${ }^{29}$ Briefly, $5 \times 10^{5}$ nucleated cells from bone marrow following red blood cell lysis, were plated into $25 \mathrm{~cm}^{2}$ culture flask in triplicates containing $6 \mathrm{~mL}$ a-MEM medium with lot-selected non-heat-inactivated $20 \%$ FBS and $0.1 \mathrm{mmol} \cdot \mathrm{L}^{-1}$ of $\beta$-mercaptoethanol. Cells were incubated for 14 days at $37^{\circ} \mathrm{C}$ in a humidified atmosphere with $5 \% \mathrm{CO}_{2}$. Cells were washed with PBS, fixed with methanol and stained with $0.5 \%$ crystal violet. Only colonies consisting 50 or more cells are used for determining colony forming efficiency.

\section{FACS}

For isolating preosteoclasts to measure Gata1 mRNA levels, bone marrow cells after red blood cell lysis were incubated with $0.5 \mu \mathrm{g}$ of anti-mouse CD16/CD32 antibody on ice for $5 \mathrm{~min}$ for blocking Fc receptors. Cells were stained for the following mouse antibodies; mouse CD45-APC, CD14-FITC, CD11b-Alexa-780, and $M-C S F$ receptor-PE conjugated antibodies (ThermoFisher; Waltham, MA, USA) according to the manufacturer's directions. Cells were sorted using FACS calibur flow cytometer (BD Bioscience) and osteoclasts were identified as $\mathrm{CD}_{4} 5^{+} / \mathrm{CD} 14^{+} / \mathrm{CD} 11 \mathrm{~b}^{+} /$ MSCFR $^{+}$cells.

Real-time PCR

Total RNA was extracted using RNeasy Mini kit (Qiagen, MD, USA) and treated with DNase I (Promega, WI, USA); $1-2 \mu \mathrm{g}$ was reverse transcribed using MultiScribe Reverse Transcriptase (ABI) (Thermo Fisher Scientific, MA, USA) for quantitative PCR assays. For mouse Epor analysis, Taqman primers and probe and mouse S16 primers 
and probe (house-keeping gene) were used as described previously. ${ }^{8}$ For all other PCR assays, SYBR green real-time PCR was carried out using gene specific primers (Supplementary Table 1) with normalization to house-keeping gene $\beta$-Actin using SYBR Green dye and qPCR SuperMix (Roche Applied Science, IN, USA); relative mRNA quantification was calculated by delta-delta $\mathrm{Ct}$ method.

Ectopic bone formation assays with BMP2

Eight-week-old Tg6 mice and littermate controls, $\Delta$ Epo $_{\mathrm{E}^{-}}$and wild-type mice were transplanted subcutaneously with $5 \mu \mathrm{g}$ of BMP2 (\#34-8507-85, Ebioscience, CA, USA) soaked into gelatin sponge cubes $(7 \times 5 \times 5 \mathrm{~mm})$ (Gelfoam ${ }^{\mathrm{T}}$, Pfizer, NY, USA). After 4 weeks ectopic bone formation was confirmed using $x$-ray analysis (In vivo Xtreme, Bruker) and transplants were harvested. After $24 \mathrm{~h}$ fixation in $10 \%$ neutral buffered formalin, transplants were moved to PBS and analyzed by micro-CT. Following decalcification in $0.05 \mathrm{~mol} \cdot \mathrm{L}^{-1}$ EDTA, H\&E staining, and TRAP staining procedures were performed.

\section{Ectopic bone formation assays with BMSCs}

Bone marrow harvested from 8-week old Tg6- and littermate controls, $\Delta \mathrm{EpoR}_{\mathrm{E}}$-, and wild-type mice, was made into a single cell suspension and plated in $6 \mathrm{~mL}$ a-MEM medium with lot-selected nonheat inactivated $20 \%$ FBS in a $25 \mathrm{~cm}^{2}$ flask and incubated undisturbed for 7 days. The adherent cell layer was washed with PBS, treated with $1 \mathrm{mg} \cdot \mathrm{mL}^{-1}$ filter sterilized collagenase II solution for $15 \mathrm{~min}$. The monolayer was washed again with PBS and incubated with $1 \%$ trypsin for 5 min. Cells were dislodged using a sterile cell scraper and plated in a $75 \mathrm{~cm}^{2}$ flask with a-MEM medium with lot-selected nonheat inactivated 20\% FBS and incubated until confluent. Cells were passed into a $175 \mathrm{~cm}^{2}$ flask with a-MEM medium with lot-selected nonheat inactivated $20 \%$ FBS and grown until confluent. SSCs/BMSCs were separated from contaminating hematopoietic cells by negative selection using CD45 and CD11b magnetic beads (Miltenyi Biotec, CA, USA). For each transplant, 2 million BMSCs were suspended in $20 \mu \mathrm{L}$ culture medium and absorbed into $7 \times 5 \times 5 \mathrm{~mm}$ gelatin sponge cubes (Gelfoam $^{\text {TM }}$, Pfizer, NY, USA) and implanted subcutaneously in the back of 8-week-old female immunocompromised NSG mice (NOD. Cg-Prkdcscidll2rgtm1Wjl/SzJ; Jackson Laboratory, ME, USA) to form ectopic bone with marrow. For determining hematocrits, blood was collected from tail vein of mice using heparin coated capillary tubes, centrifuged in a micro-hematocrit centrifuge (Unico, Dayton, NJ, USA). Hematocrit was measured using a VIN micro-hematocrit capillary tube reader (Veterinary Information Network Bookstore, Davis, CA, USA). After 8 weeks post transplantation, ectopic bone formation was confirmed by $\mathrm{x}$-ray monitoring and transplants were harvested, fixed in 10\% neutral buffered formalin. Transplants were moved to PBS and analyzed by micro-CT. For histology, fixed transplants were decalcified in $0.05 \mathrm{~mol} \cdot \mathrm{L}^{-1}$ EDTA in PBS for 2-3 weeks, paraffin embedded, and sectioned. H\&E staining and TRAP staining were performed on sections to assess histology and to detect osteoclasts, respectively. Images were analyzed using ImageJ software to determine adipocyte and marrow area relative to total tissue area.

Serum analysis and hematocrit measurement

Blood was collected terminally by cardiac puncture and levels of mouse EPO and human EPO in serum were measured using Quantikine ELISA assays (mouse EPO \#MEPO0B; human EPO \#DEP00, R\&D Biosystems, MN, USA) according to manufacturer's instructions. Mouse $\mathrm{C}$-reactive protein in serum was measured using ELISA assay (\#ab157712, Abcam, Cambridge MA, USA). For hematocrit, blood was collected from the tail vein in heparin coated capillary tubes. The tubes were centrifuged using a microhematocrit centrifuge (Unico, NJ, USA) and hematocrits were measured using a VIN micro-hematocrit capillary tube reader (Veterinary Information Network Bookstore, CA, USA).

Statistical analysis

Data are expressed as mean \pm standard deviation. Two-tailed nonpaired Student's $t$ test was used to determine statistical significance between control and test groups. Comparisons among multiple groups were evaluated using one way analysis of variance with Dunnet's multiple comparison post hoc tests at a $=0.05$ (Graphpad Prism 6).

\section{ACKNOWLEDGEMENTS}

This work was supported by the Intramural Research Programs of the National Institute of Diabetes and Digestive and Kidney Diseases and of the National Institute of Dental and Craniofacial Research at the National Institutes of Health.

\section{AUTHOR CONTRIBUTIONS}

S.S. designed the research, performed the experiments, analyzed the data, and prepared the manuscript, L.F.d.C. designed the research, performed the experiments, and analyzed the data, S.D. designed the research and performed the experiments, P. G.R. and C.T.N. designed the research, analyzed the data, and prepared the manuscript.

\section{ADDITIONAL INFORMATION}

The online version of this article (https://doi.org/10.1038/s41413-019-0060-0) contains supplementary material, which is available to authorized users.

Competing interests: The authors declare no competing interests.

\section{REFERENCES}

1. Zhang, Y. et al. Erythropoietin action in stress response, tissue maintenance and metabolism. Int. J. Mol. Sci. 15, 10296-10333 (2014).

2. Lin, C. S., Lim, S. K., D'Agati, V. \& Costantini, F. Differential effects of an erythropoietin receptor gene disruption on primitive and definitive erythropoiesis. Genes Dev. 10, 154-164 (1996).

3. Wu, H., Liu, X., Jaenisch, R. \& Lodish, H. F. Generation of committed erythroid BFU$\mathrm{E}$ and CFU-E progenitors does not require erythropoietin or the erythropoietin receptor. Cell 83, 59-67 (1995).

4. Jelkmann, W. Physiology and pharmacology of erythropoietin. Transfus. Med. Hemother 40, 302-309 (2013).

5. Beleslin-Cokic, B. B. et al. Erythropoietin and hypoxia stimulate erythropoietin receptor and nitric oxide production by endothelial cells. Blood 104, 2073-2080 (2004).

6. $\mathrm{Yu}, \mathrm{X}$. et al. Erythropoietin receptor signalling is required for normal brain development. Development 129, 505-516 (2002).

7. Ogilvie, M. et al. Erythropoietin stimulates proliferation and interferes with differentiation of myoblasts. J. Biol. Chem. 275, 39754-39761 (2000).

8. Teng, R. et al. Disrupted erythropoietin signalling promotes obesity and alters hypothalamus proopiomelanocortin production. Nat. Commun. 2, 520 (2011).

9. Choi, D. et al. Erythropoietin protects against diabetes through direct effects on pancreatic beta cells. J. Exp. Med. 207, 2831-2842 (2010).

10. Bianco, P., Riminucci, M., Gronthos, S. \& Robey, P. G. Bone marrow stromal stem cells: nature, biology, and potential applications. Stem Cells 19, 180-192 (2001).

11. James, A. W. Review of signaling pathways governing MSC osteogenic and adipogenic differentiation. Sci. (Cairo) 2013, 684736 (2013).

12. Schwartz, A. V. et al. Vertebral bone marrow fat associated with lower trabecular BMD and prevalent vertebral fracture in older adults. J. Clin. Endocrinol. Metab. 98, 2294-2300 (2013).

13. Shen, W. et al. MRI-measured pelvic bone marrow adipose tissue is inversely related to DXA-measured bone mineral in younger and older adults. Eur. J. Clin. Nutr. 66, 983-988 (2012).

14. Justesen, J. et al. Adipocyte tissue volume in bone marrow is increased with aging and in patients with osteoporosis. Biogerontology 2, 165-171 (2001).

15. Compston, J. E. et al. Obesity, health-care utilization, and health-related quality of life after fracture in postmenopausal women: global longitudinal study of osteoporosis in women (GLOW). Calcif. Tissue Int. 94, 223-231 (2014).

16. Sheu, Y. et al. Vertebral bone marrow fat, bone mineral density and diabetes: The Osteoporotic Fractures in Men (MrOS) study. Bone 97, 299-305 (2017).

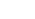


17. Crockett, J. C., Rogers, M. J., Coxon, F. P., Hocking, L. J. \& Helfrich, M. H. Bone remodelling at a glance. J. Cell Sci. 124, 991-998 (2011).

18. Shiozawa, Y. et al. Erythropoietin couples hematopoiesis with bone formation. PLOS ONE 5, e10853 (2010).

19. Singbrant, S. et al. Erythropoietin couples erythropoiesis, B-lymphopoiesis, and bone homeostasis within the bone marrow microenvironment. Blood 117, 5631-5642 (2011).

20. Wan, L. et al. EPO promotes bone repair through enhanced cartilaginous callus formation and angiogenesis. PloS ONE 9, e102010 (2014).

21. Chen, S., Li, J., Peng, H., Zhou, J. \& Fang, H. Administration of erythropoietin exerts protective effects against glucocorticoid-induced osteonecrosis of the femoral head in rats. Int. J. Mol. Med. 33, 840-848 (2014).

22. Hiram-Bab, S. et al. Erythropoietin directly stimulates osteoclast precursors and induces bone loss. FASEB J. 29, 1890-1900 (2015).

23. Ruschitzka, F. T. et al. Nitric oxide prevents cardiovascular disease and determines survival in polyglobulic mice overexpressing erythropoietin. Proc. Natl Acad. Sci. USA 97, 11609-11613 (2000).

24. Suzuki, N. et al. Erythroid-specific expression of the erythropoietin receptor rescued its null mutant mice from lethality. Blood 100, 2279-2288 (2002).

25. Alnaeeli, M. et al. Erythropoietin signaling: a novel regulator of white adipose tissue inflammation during diet-induced obesity. Diabetes 63, 2415-2431 (2014).

26. Tachi, K. et al. Enhancement of bone morphogenetic protein-2-induced ectopic bone formation by transforming growth factor-beta1. Tissue Eng. Part A 17, 597-606 (2011).

27. Hata, K. et al. Differential roles of Smad1 and p38 kinase in regulation of peroxisome proliferator-activating receptor gamma during bone morphogenetic protein 2-induced adipogenesis. Mol. Biol. Cell 14, 545-555 (2003).

28. Jin, W. et al. Schnurri-2 controls BMP-dependent adipogenesis via interaction with Smad proteins. Dev. Cell 10, 461-471 (2006).

29. Nemeth, K., Mayer, B., Sworder, B. J., Kuznetsov, S. A. \& Mezey, E. A practical guide to culturing mouse and human bone marrow stromal cells. Curr. Protoc. Immunol. 102, 22F.12 (2013).

30. Anjos-Afonso, F. \& Bonnet, D. Isolation, culture, and differentiation potential of mouse marrow stromal cells. Curr. Protoc. Stem Cell Biol. (2008). (Chapter 2, Unit 2B.3.1-2B.3.11).

31. Robey, P. G., Kuznetsov, S. A., Riminucci, M. \& Bianco, P. Bone marrow stromal cell assays: in vitro and in vivo. Methods Mol. Biol. 1130, 279-293 (2014).

32. Tong, Q., Tsai, J., Tan, G., Dalgin, G. \& Hotamisligil, G. S. Interaction between GATA and the C/EBP family of transcription factors is critical in GATA-mediated suppression of adipocyte differentiation. Mol. Cell. Biol. 25, 706-715 (2005).

33. Zhang, Y., Rogers, H. M., Zhang, X. \& Noguchi, C. T. Sex difference in mouse metabolic response to erythropoietin. FASEB J. 31, 2661-2673 (2017).

34. Glatt, V., Canalis, E., Stadmeyer, L. \& Bouxsein, M. L. Age-related changes in trabecular architecture differ in female and male C57BL/6J mice. J. Bone Miner. Res. 22, 1197-1207 (2007).

35. Shu, L. et al. High-fat diet causes bone loss in young mice by promoting osteoclastogenesis through alteration of the bone marrow environment. Calcif. Tissue Int. 96, 313-323 (2015).

36. Cohen, A. et al. Abdominal fat is associated with lower bone formation and inferior bone quality in healthy premenopausal women: a transiliac bone biopsy study. J. Clin. Endocrinol. Metab. 98, 2562-2572 (2013).

37. Charles, J. F. et al. Inflammatory arthritis increases mouse osteoclast precursors with myeloid suppressor function. J. Clin. Investig. 122, 4592-4605 (2012).

38. Cao, J. J., Gregoire, B. R. \& Shen, C. L. A high-fat diet decreases bone mass in growing mice with systemic chronic inflammation induced by low-dose, slowrelease lipopolysaccharide pellets. J. Nutr. 147, 1909-1916 (2017).

39. Foskett, A., Alnaeeli, M., Wang, L., Teng, R. \& Noguchi, C. T. The effects of erythropoietin dose titration during high-fat diet-induced obesity. J. Biomed. Biotechnol. 2011, 373781 (2011).

40. Griffith, J. F. et al. Bone marrow fat content in the elderly: a reversal of sex difference seen in younger subjects. J. Magn. Reson. Imaging. 36, 225-230 (2012).

41. Rauner, M. et al. Increased EPO levels are associated with bone loss in mice lacking PHD2 in EPO-producing cells. J. Bone Min. Res 31, 1877-1887 (2016).
42. Kugimiya, F. et al. Involvement of endogenous bone morphogenetic protein (BMP) 2 and BMP6 in bone formation. J. Biol. Chem. 280, 35704-35712 (2005).

43. Champagne, C. M., Takebe, J., Offenbacher, S. \& Cooper, L. F. Macrophage cell lines produce osteoinductive signals that include bone morphogenetic protein-2. Bone 30, 26-31 (2002).

44. Vukicevic, S. \& Grgurevic, L. BMP-6 and mesenchymal stem cell differentiation. Cytokine Growth Factor Rev. 20, 441-448 (2009).

45. Mi, M. et al. Chondrocyte BMP2 signaling plays an essential role in bone fracture healing. Gene 512, 211-218 (2013).

46. Lifshitz, L., Tabak, G., Gassmann, M., Mittelman, M. \& Neumann, D. Macrophages as novel target cells for erythropoietin. Haematologica 95, 1823-1831 (2010).

47. Patel, J. J. et al. Dual delivery of EPO and BMP2 from a novel modular polyvarepsilon-caprolactone construct to increase the bone formation in prefabricated bone flaps. Tissue Eng. Part C. Methods 21, 889-897 (2015).

48. Sun, H., Jung, Y., Shiozawa, Y., Taichman, R. S. \& Krebsbach, P. H. Erythropoietin modulates the structure of bone morphogenetic protein 2-engineered cranial bone. Tissue Eng. Part A 18, 2095-2105 (2012).

49. Tong, Q. et al. Function of GATA transcription factors in preadipocyte-adipocyte transition. Science 290, 134-138 (2000).

50. Rankin, E. B. et al. The HIF signaling pathway in osteoblasts directly modulates erythropoiesis through the production of EPO. Cell 149, 63-74 (2012).

51. Rolfing, J. H. et al. The osteogenic effect of erythropoietin on human mesenchymal stromal cells is dose-dependent and involves nonhematopoietic receptors and multiple intracellular signaling pathways. Stem Cell Rev. 10, 69-78 (2014).

52. Kim, J. et al. Erythropoietin mediated bone formation is regulated by mTOR signaling. J. Cell Biochem. 113, 220-228 (2012).

53. Li, C. J. et al. MicroRNA-188 regulates age-related switch between osteoblast and adipocyte differentiation. J. Clin. Investig. 125, 1509-1522 (2015).

54. Sui, B. et al. Mesenchymal progenitors in osteopenias of diverse pathologies: differential characteristics in the common shift from osteoblastogenesis to adipogenesis. Sci. Rep. 6, 30186 (2016).

55. Jing, $\mathrm{H}$. et al. Suppression of EZH2 prevents the shift of osteoporotic MSC fate to adipocyte and enhances bone formation during osteoporosis. Mol. Ther. 24, 217-229 (2016).

56. Tavassoli, M. Ultrastructural development of bone marrow adipose cell. Acta Anat. (Basel) 94, 65-77 (1976).

57. Suchacki, K. J., Cawthorn, W. P. \& Rosen, C. J. Bone marrow adipose tissue: formation, function and regulation. Curr. Opin. Pharm. 28, 50-56 (2016).

58. Gao, Y. et al. Magnetic resonance imaging-measured bone marrow adipose tissue area is inversely related to cortical bone area in children and adolescents aged 5-18 years. J. Clin. Densitom. 18, 203-208 (2015).

59. Scheller, E. L. et al. Use of osmium tetroxide staining with microcomputerized tomography to visualize and quantify bone marrow adipose tissue in vivo. Methods Enzym. 537, 123-139 (2014).

60. Bakker, A. D. \& Klein-Nulend, J. Osteoblast isolation from murine calvaria and long bones. Methods Mol. Biol. 816, 19-29 (2012).

Open Access This article is licensed under a Creative Commons Attribution 4.0 International License, which permits use, sharing, adaptation, distribution and reproduction in any medium or format, as long as you give appropriate credit to the original author(s) and the source, provide a link to the Creative Commons license, and indicate if changes were made. The images or other third party material in this article are included in the article's Creative Commons license, unless indicated otherwise in a credit line to the material. If material is not included in the article's Creative Commons license and your intended use is not permitted by statutory regulation or exceeds the permitted use, you will need to obtain permission directly from the copyright holder. To view a copy of this license, visit http://creativecommons. org/licenses/by/4.0/.

(c) The Author(s) 2019 